\title{
Lump and Interaction Solutions to the $(3+1)$-Dimensional Variable-Coefficient Nonlinear Wave Equation with Multidimensional Binary Bell Polynomials
}

\author{
Xuejun Zhou $\mathbb{D}^{1},{ }^{1}$ Onur Alp Ilhan $\mathbb{D}^{\mathbb{D}},{ }^{2}$ Fangyuan Zhou ${ }^{\mathbb{D}},{ }^{1}$ Sutarto Sutarto $\mathbb{D}{ }^{3}$ \\ Jalil Manafian $\left(\mathbb{D},{ }^{4,5}\right.$ and Mostafa Abotaleb $\mathbb{1}^{6}$ \\ ${ }^{1}$ College of Physics and Electronic Information, Yan'an University, Yan'an, Shanxi 716000, China \\ ${ }^{2}$ Department of Mathematics, Faculty of Education, Erciyes University, 38039, Melikgazi, Kayseri, Turkey \\ ${ }^{3}$ Mathematics Education, Faculty of Science, Engineering and Applied, Universitas Pendidikan Mandalika, Indonesia \\ ${ }^{4}$ Department of Applied Mathematics, Faculty of Mathematical Sciences, University of Tabriz, Tabriz, Iran \\ ${ }^{5}$ Natural Sciences Faculty, Lankaran State University, 50, H. Aslanov Str., Lankaran, Azerbaijan \\ ${ }^{6}$ Department of System Programming, South Ural State University, 454080 Chelyabinsk, Russia \\ Correspondence should be addressed to Jalil Manafian; j_manafianheris@tabrizu.ac.ir
}

Received 4 September 2021; Accepted 11 November 2021; Published 13 December 2021

Academic Editor: Giovanni Di Fratta

Copyright ( 92021 Xuejun Zhou et al. This is an open access article distributed under the Creative Commons Attribution License, which permits unrestricted use, distribution, and reproduction in any medium, provided the original work is properly cited.

In this paper, we study the $(3+1)$-dimensional variable-coefficient nonlinear wave equation which is taken in soliton theory and generated by utilizing the Hirota bilinear technique. We obtain some new exact analytical solutions, containing interaction between a lump-two kink solitons, interaction between two lumps, and interaction between two lumps-soliton, lump-periodic, and lump-three kink solutions for the generalized $(3+1)$-dimensional nonlinear wave equation in liquid with gas bubbles by the Maple symbolic package. Making use of Hirota's bilinear scheme, we obtain its general soliton solutions in terms of bilinear form equation to the considered model which can be obtained by multidimensional binary Bell polynomials. Furthermore, we analyze typical dynamics of the high-order soliton solutions to show the regularity of solutions and also illustrate their behavior graphically.

\section{Introduction}

It is known that there are a variety of useful and powerful tools to deal with the nonlocal equations, namely, the improved $\tan (\phi / 2)$-expansion method [1], the homotopy perturbation method [2], Lie symmetry analysis [3], the Bäcklund transformation method [4], the sine-Gordon expansion approach [5], the $\left(G^{\prime} / G, 1 / G\right)$, modified $\left(G^{\prime} / G^{2}\right)$, and $\left(1 / G^{\prime}\right)$-expansion methods [6], the multiple Expfunction method [7-10], Hirota's bilinear method including the $(2+1)$-dimensional variable-coefficient Caudrey-DoddGibbon-Kotera-Sawada equation [11], the generalized unstable space time fractional nonlinear Schrödinger equation [12], the inverse Cauchy problems [13], a generalized hyper- elastic rod equation [14], the Kadomtsev-Petviashvili equation [15], the bKP equation [16], the generalized Burgers equation [16], the inverse scattering transformation method $[17,18]$, and the KP hierarchy reduction method [19]. Combining Hirota's bilinear method with the KP reduction hierarchy method, very recently, Rao et al. [20] discussed the Kadomtsev-Petviashvili-based system and studied the fusion of lumps and line solitons into line solitons, fission of line solitons into lumps and line solitons, a mixture of fission and fusion processes of lumps and line solitons, and the inelastic collision of line rogue waves and line soliton. An improved Hirota bilinear method for the nonlocal complex MKdV equation was constructed in Ref. [21]. Sun et al. [22] investigated a generalized three-component 
Hirota-Satsuma coupled KdV equation describing the interactions of two long waves with different dispersion relations by applying Hirota bilinear operator theory. By employing the Hirota bilinear method, Ma [23] constructed the $N$-soliton solution for three integrable equations in $(2+1)$-dimensions including the $(2+1)$-dimensional $\mathrm{KdV}$ equation, the Kadomtsev-Petviashvili equation, and the $(2+1)$-dimensional Hirota-Satsuma-Ito equation and gave the asymptotic analysis of $N$-soliton solution. Also, by using the bilinear Bell polynomial approach, Cui [24] obtained some new exact solutions for the new extended $(2+1)$-dimensional Boussinesq equation which can be applied to describe the propagation of shallow water waves. And in Ref. [25], Cheng and coauthors obtained the velocity resonance mechanism and the two-, three-, and four-soliton molecules by utilizing the Hirota bilinear method for the combined $(2 N+1)$ th-order Lax's KdV equation. In [26], the new solitary wave solutions for the $(3+1)$-dimensional extended Jimbo-Miwa equations were investigated. Moreover, Ma obtained lump solutions to a combined fourth-order nonlinear PDE in $(2+1)$-dimensions [27] and interaction solutions to the Hirota-SatsumaIto equation in $(2+1)$-dimensions [28].

Consider the $(3+1)$-dimensional variable-coefficient nonlinear wave equation [29-31] which will be investigated below:

$\left(u_{t}+\phi_{1}(t) u u_{x}+\phi_{2}(t) u_{x x x}+\phi_{3}(t) u_{x}\right)_{x}+\phi_{4}(t) u_{y y}+\phi_{5}(t) u_{z z}=0$,

where $u=u(x, y, z, t)$ is an unknown function and $\phi_{j}(t)$ $(j=1, \cdots, 5)$ are all optional amounts. And $u$ is the wave amplitude, the variable coefficients $\phi_{1}(t), \phi_{2}(t), \phi_{3}(t), \phi_{4}(t)$, and $\phi_{5}(t)$ denote the bubble-liquid nonlinearity, the bubble-liquid dispersion, the bubble-liquid viscosity, the $y$ -transverse-perturbation, and the $z$-transverse-perturbation, respectively, and they are all real functions of $t$. Equation (1) can be transformed to the following:

(i) The well-known constant-coefficient KadomtsevPetviashvili equation with $\phi_{1}(t)= \pm 6, \phi_{2}(t)=1, \phi_{4}(t)$ $=3, \phi_{3}(t)=\phi_{5}(t)=0$ as

$$
\left(u_{t} \pm 6 u u_{x}+u_{x x x}\right)_{x}+3 u_{y y}=0
$$

(ii) The cylindrical $\mathrm{KdV}$ equation with $\phi_{1}(t)= \pm 6, \phi_{2}(t)$ $=1, \phi_{3}(t)=1 / 2 t, \phi_{4}(t)=\phi_{5}(t)=0$ as

$$
u_{t} \pm 6 u u_{x}+u_{\mathrm{x} x x}+\frac{1}{2 t} u_{x}=0
$$

For equation (1), some solutions including the multisoliton, Bäcklund transformation, infinite conservation laws, lump solutions, and other soliton wave solutions have been investigated in Refs. [29, 30]. Also, Guo and Chen [31] studied equation (1) and obtained the multisoliton solutions and periodic solutions including $X$-periodic, $Y$-periodic, and 2 -periodic wave solutions. The solitons, periodic, and travelling waves of a generalized $(3+1)$-dimensional variablecoefficient nonlinear wave equation in liquid with gas bubbles were caught by Deng and Gao [29]. In [32], the first-order lump wave solution and second-order lump wave solution according to the corresponding two-soliton solution and four-soliton solution were presented. The multisoliton solutions and periodic solutions for the $(3+1$ )-dimensional variable-coefficient nonlinear wave equation in liquid with gas bubbles were reported by Guo and Chen [31]. Two different types of bright solutions for the generalized $(3+1)$-dimensional nonlinear wave equation by the traveling wave method were obtained by Guo and Chen [33]. The special $N$ wave solutions by applying the linear superposition principle, the resonant multiple wave solutions, and complexiton solutions were investigated for the generalized $(3+1)$-dimensional nonlinear wave in liquid with gas bubbles in [34]. Ma and coauthors probed and analyzed $\mathrm{N}$-soliton solutions and the Hirota conditions in $(1+1)$-dimensions [13] and $(1+2)$-dimensions [35].

During the last years, the various analytical methods were developed to find the exact solutions by powerful scholars for interesting fields of research because of their wide number of applications in the engineering and manufacturing fields, nonlinear models, for example, nonlinear Schrodinger equation [36], the conformable nonlinear differential equation governing wave-propagation in low-pass electrical transmission lines [37], the $(2+1)$-dimensional coupled variant Boussinesq equations [38], the nonlinear directional couplers with metamaterials by including spatial-temporal fractional beta derivative evolution [39], a new $(3+1)$-dimensional Hirota bilinear equation [40], oblique resonant nonlinear waves with dual-power law nonlinearity [41], the coupled Schrödinger-Boussinesq system with the beta derivative [42], and the Hirota-Maccari system [43].

The major aim of this paper is to obtain some novel exact analytical solutions, including interaction between a lump-two kink solitons, interaction between two lumps, and interaction between two lumps-soliton, lump-periodic, and lump-three kink solutions for the $(3+1)$-dimensional variable-coefficient (VC) nonlinear wave equation in liquid with gas bubbles through the method of the bilinear analysis.

The rest of this article is organized as follows. In Section 2, explanations of multidimensional binary Bell polynomials are given. Also, in Section 3, the bilinear form equation to the $(3+1)$-dimensional VC nonlinear wave equation is constructed. In Section 4, we obtain the interaction between a lump-two kink solitons, interaction between two lumps, and interaction between two lumps-soliton, lump-periodic, and lump-three kink solutions along with depicting 3D, density, and 2D graphs for the VC nonlinear wave equation. The conclusion is given in Section 5 .

\section{Multidimensional Binary Bell Polynomials}

Based on Ref. [16, 44, 45], consider $\xi=\xi\left(x_{1}, x_{2}, \cdots, x_{n}\right)$ a $C^{\infty}$ function with multivariables; the polynomials of the following form 


$$
Y_{n_{1} x_{1}, \cdots, n_{j} x_{j}}(\xi) \equiv Y_{n_{1}, \cdots, n_{j}}\left(\xi_{s_{1} x_{1}, \cdots, s_{j} x_{j}}\right)=e^{-\xi} \partial_{x_{1}}^{n_{1}} \cdots \partial_{x_{j}}^{n_{j}} \xi^{\xi}
$$

are called the multidimensional Bell polynomials:

$$
\xi_{s_{1} x_{1}, \cdots, s_{j} x_{j}}=\partial_{x_{1}}^{s_{1}} \cdots \partial_{x_{j}}^{s_{j}} \xi, \xi_{0 x_{i}} \equiv \xi, s_{1}=0, \cdots, n_{1} ; \cdots ; s_{j}=0, \cdots, n_{j},
$$

and we have

$$
\begin{aligned}
& Y_{1}(\xi)=\xi_{x}, Y_{2}(\xi)=\xi_{2 x}+\xi_{x}^{2}, Y_{3}(\xi)=\xi_{3 x}+3 \xi_{x} \xi_{2 x}+\xi_{x}^{3}, \cdots, \xi=\xi(x, t), \\
& Y_{x, t}(\xi)=\xi_{x, t}+\xi_{x} \xi_{t}, Y_{2 x, t}(\xi)=\xi_{2 x, t}+\xi_{2 x} \xi_{t}+2 \xi_{x, t} \xi_{x}+\xi_{x}^{2} \xi_{t}, \cdots
\end{aligned}
$$

The multidimensional binary Bell polynomials can be written as

$$
\Sigma_{n_{1} x_{1}, \cdots, n_{j} x_{j}}(\alpha, \beta)=\left.Y_{n_{1}, \cdots, n_{j}}(\xi)\right|_{\xi_{s_{1} x_{1}, \cdots, s_{j} x_{j}}=} \begin{cases}\alpha_{s_{1} x_{1}, \cdots, s_{j} x_{j}} & s_{1}+s_{2}+\cdots+s_{j} \text { is odd } \\ \beta_{s_{1} x_{1}, \cdots, s_{j} x_{j}}, & s_{1}+s_{2}+\cdots+s_{j} \text { is even. }\end{cases}
$$

We have the following conditions as

$$
\Sigma_{x}(\alpha)=\alpha_{x}, \Sigma_{2 x}(\alpha, \beta)=\beta_{2 x}+\alpha_{x}^{2}, \Sigma_{x, t}(\alpha, \beta)=\beta_{x, t}+\alpha_{x} \alpha_{t}, \cdots .
$$

Proposition 1. Suppose $\alpha=\ln (\Theta / \Delta), \beta=\ln (\Theta \Delta)$, then the relations between binary Bell polynomials and Hirota $D$ -operator can be written as below:

$$
\left.\Sigma_{n_{1} x_{1}, \cdots, n_{j} x_{j}}(\alpha, \beta)\right|_{\alpha=\ln (\Theta / \Delta), \beta=\ln (\Theta \Delta)}=(\Theta \Delta)^{-1} D_{x_{1}}^{n_{1}} \cdots D_{x_{j}}^{n_{j}} \Theta \Delta,
$$

with Hirota operator

$$
\prod_{i=1}^{j} D_{x_{i}}^{n_{i}} g \cdot \eta=\left.\prod_{i=1}^{j}\left(\frac{\partial}{\partial x_{i}}-\frac{\partial}{\partial x_{i}{ }^{\prime}}\right)^{n_{i}} \Theta\left(x_{1}, \cdots, x_{j}\right) \Delta\left(x_{1}{ }^{\prime}, \cdots, x_{j}{ }^{\prime}\right)\right|_{x_{1}=x_{1}{ }^{\prime}, \cdots, x_{j}=x_{j}^{\prime}} .
$$

Proposition 2. Take $\Xi(\gamma)=\sum_{i} \delta_{i} \mathfrak{P}_{s_{1} x_{1}, \cdots, s_{j} x_{j}}=0$ and $\alpha=\ln$ $(\Theta / \Delta), \beta=\ln (\Theta \Delta)$, we have

$$
\left\{\begin{array}{l}
\sum_{i} \delta_{1 i} Y_{n_{1} x_{1}, \cdots, n_{j} x_{j}}(\alpha, \beta)=0, \\
\sum_{i} \delta_{1 i} Y_{s_{1} x_{1}, \cdots, s_{j} x_{j}}(\alpha, \beta)=0,
\end{array}\right.
$$

which need to satisfy

$$
\mathfrak{R}\left(\gamma^{\prime}, \gamma\right)=\mathfrak{R}\left(\gamma^{\prime}\right)-\mathfrak{R}(\gamma)=\mathfrak{R}(\beta+\alpha)-\mathfrak{R}(\beta-\alpha)=0 .
$$

The generalized Bell polynomials $Y_{n_{1} x_{1}, \cdots, n_{j} x_{j}}(\xi)$ are as

$$
\begin{aligned}
& (\Theta \Delta)^{-1} D_{x_{1}}^{n_{1}} \cdots D_{x_{j}}^{n_{j}} \Theta \Delta=\left.\sum_{n_{1} x_{1}, \cdots, n_{j} x_{j}}(\alpha, \beta)\right|_{\alpha=\ln (\Theta / \Delta), \beta=\ln (\Theta \Delta)} \\
& \quad=\left.\sum_{n_{1} x_{1}, \cdots, n_{j} x_{j}}(\alpha, \alpha+\gamma)\right|_{\alpha=\ln (\Theta / \Delta), \gamma=\ln (\Theta \Delta)} \\
& \quad=\sum_{k_{1}}^{n_{1}} \cdots \sum_{k_{j}}^{n_{j}} \prod_{i=1}^{j}\left(\begin{array}{c}
n_{i} \\
k_{i}
\end{array}\right) \mathfrak{P}_{k_{1} x_{1}, \cdots, k_{j} x_{j}}(\gamma) Y_{\left(n_{1}-k_{1}\right) x_{1}, \cdots,\left(n_{j}-k_{j}\right) x_{j}}(\alpha) .
\end{aligned}
$$

The Cole-Hopf transformation will be as

$$
\begin{gathered}
Y_{k_{1} x_{1}, \cdots, k_{j} x_{j}}(\alpha=\ln (\varphi))=\frac{\varphi_{n_{1} x_{1}, \cdots, n_{j} x_{j}}}{\varphi}, \\
\left.(\Theta \Delta)^{-1} D_{x_{1}}^{n_{1}} \cdots D_{x_{j}}^{n_{j}} \Theta \Delta\right|_{\Delta=\exp (\gamma / 2), \Theta / \Delta=\varphi} \\
=\varphi^{-1} \sum_{k_{1}}^{n_{1}} \cdots \sum_{k_{j}}^{n_{j}} \prod_{d=1}^{j}\left(\begin{array}{c}
n_{d} \\
k_{d}
\end{array}\right) \mathfrak{P}_{k_{1} x_{1}, \cdots, k_{d} x_{d}}(\gamma) \varphi_{\left(n_{1}-k_{1}\right) x_{1}, \cdots,\left(n_{d}-k_{d}\right) x_{d}},
\end{gathered}
$$

with

$$
\begin{aligned}
Y_{t}(\alpha) & =\frac{\varphi_{t}}{\varphi} \\
Y_{2 x}(\alpha, \beta) & =\gamma_{2 x}+\frac{\varphi_{2 x}}{\varphi} \\
Y_{2 x, y}(\alpha, \beta) & =\frac{\gamma_{2 x} \varphi_{y}}{\varphi}+\frac{2 \gamma_{x, y} \varphi_{x}}{\varphi}+\frac{\varphi_{2 x, y}}{\varphi} .
\end{aligned}
$$

\section{Bilinear Form Equation to the $(3+1)$ D VC Nonlinear Wave Equation}

To find the linearizing representation, we consider the below:

$$
\begin{aligned}
& u=c \gamma_{x x}+u_{0}, \\
& \gamma=\gamma(x, y, z, t), \\
& c=c(t) .
\end{aligned}
$$

Inserting equation (16) into equation (1), one obtains

$$
\begin{aligned}
\Re(\gamma)= & \left(\frac{d}{d t} c(t)\right) \frac{\partial}{\partial x} \gamma(x, y, z, t) \\
& +c(t) \frac{\partial^{2}}{\partial x \partial t} \gamma(x, y, z, t)+\phi_{1}(t)(c(t))^{2}\left(\frac{\partial}{\partial x} \gamma(x, y, z, t)\right)^{2} \\
& +\phi_{1}(t)(c(t))^{2} \gamma(x, y, z, t) \frac{\partial^{2}}{\partial x^{2}} \gamma(x, y, z, t) \\
& +\phi_{2}(t) c(t) \frac{\partial^{4}}{\partial x^{4}} \gamma(x, y, z, t)+\phi_{3}(t) c(t) \frac{\partial^{2}}{\partial x^{2}} \gamma(x, y, z, t) \\
& +\phi_{4}(t) c(t) \frac{\partial^{2}}{\partial y^{2}} \gamma(x, y, z, t)+\phi_{5}(t) c(t) \frac{\partial^{2}}{\partial z^{2}} \gamma(x, y, z, t)=0
\end{aligned}
$$

with 


$$
c=12 \text {. }
$$

The new equation $\mathfrak{R}(\gamma)$ is as

$\mathfrak{R}(\gamma)=\mathfrak{P}_{x, t}+\phi_{2}(t)\left(\mathfrak{P}_{4 x}+3 \mathfrak{P}_{2 x}^{2}\right)+\phi_{3}(t) \mathfrak{P}_{2 x}+\phi_{4}(t) \mathfrak{P}_{2 y}+\phi_{5}(t) \mathfrak{P}_{2 z}=0$.

Applying a change of dependent variable

$$
\gamma=\ln (g) \Longleftrightarrow u=12 \ln (g)_{x x} .
$$

Theorem 3. With the following relations

$$
\gamma=\ln (g) \Longleftrightarrow u=12 \ln (g)_{x x}
$$

into equation (1), the $(3+1) D V C$ nonlinear wave equation can be linearized as the following bilinear equation:

$\mathfrak{R}(g)=\left(g g_{x t}-g_{x} g_{t}\right)+\phi_{2}(t)\left(g g_{4 x}-4 g_{x} g_{3 x}+3 g_{x x}^{2}\right)+\phi_{3}(t)\left(g g_{x x}-g_{x}^{2}\right)$,

$\phi_{4}(t)\left(g g_{y y}-g_{y}^{2}\right)+\phi_{5}(t)\left(g g_{z z}-g_{z}^{2}\right)=1 / 2\left(D_{x} D_{t}+\phi_{2}(t)\right.$ $\left.D_{x}^{4}+\phi_{3}(t) D_{x}^{2}+\phi_{4}(t) D_{y}^{2}+\phi_{5}(t) D_{z}^{2}\right) g \cdot g=0$, where $g=g(x$, $y, z, t)$ and $\gamma=\gamma(x, y, z, t)$.

\section{Lump, Lump-Kink, and Other Wave Solutions}

We would like to derive the general soliton solutions containing interaction between lump-two kink solitons, interaction between two lumps, and interaction between two lumps-soliton, lump-periodic, and lump-three kink solutions.

4.1. Interaction between a Lump-Two Kink Soliton Solutions. In this section, we would like to present the general solutions of the $(3+1)$-dimensional variable-coefficient nonlinear wave equation through utilizing the bilinear method as the below frame:

$$
\begin{aligned}
g & =\tau_{1}^{2}+\tau_{2}^{2}+\exp \left(\tau_{3}\right)+\exp \left(\tau_{4}\right)+\exp \left(\tau_{3}+\tau_{4}\right)+\varepsilon_{5}(t), \tau_{j} \\
& =\alpha_{j} x+\beta_{j} y+\delta_{j} z+\varepsilon_{j}(t), j \\
& =1,2,3, \varepsilon_{0}>0 .
\end{aligned}
$$

The values $\alpha_{i}, \beta_{i}, \delta_{i}, \varepsilon_{i}(t)(i=1,2,3)$ are real constants to be computed. By substituting (23) into (22), we obtain a system containing 42 nonlinear equations. By solving the nonlinear system, the determined coefficients will be got as the below cases.

Type I

$$
\begin{aligned}
\varepsilon_{1}(t) & =\varepsilon_{3}(t)=\varepsilon_{4}(t)=\varepsilon_{5}(t)=0, \varepsilon_{2}(t) \\
& =\int-\frac{\alpha_{2}{ }^{2} \phi_{2}(t)+\beta_{2}{ }^{2} \phi_{3}(t)}{\alpha_{2}} d t, \alpha_{1}=\alpha_{3}=\alpha_{4}=0, \alpha_{2}=\alpha_{2}, \\
\beta_{1}= & \beta_{3}=\beta_{4}=0, \beta_{2}=\beta_{2}, \delta_{1}=\delta_{1}, \delta_{2}=\delta_{2}, \delta_{3}=\delta_{3}, \delta_{4}=\delta_{4} .
\end{aligned}
$$

The solutions are given as follows:

$$
\begin{aligned}
u_{1}= & \frac{24 \alpha_{2}^{2}}{\delta_{1}{ }^{2} z^{2}+\left(\alpha_{2} x+\beta_{2} y+\delta_{2} z+\int-\alpha_{2}{ }^{2} \phi_{2}(t)+\beta_{2}{ }^{2} \phi_{3}(t) / \alpha_{2} d t\right)^{2}+e^{\delta_{3} z}+e^{\delta_{4} z}+e^{\delta_{3} z+\delta_{4} z}} \\
& -\frac{48\left(\alpha_{2} x+\beta_{2} y+\delta_{2} z+\int-\alpha_{2}{ }^{2} \phi_{2}(t)+\beta_{2}{ }^{2} \phi_{3}(t) / \alpha_{2} d t\right)^{2} \alpha_{2}{ }^{2}}{\left(\delta_{1}{ }^{2} z^{2}+\left(\alpha_{2} x+\beta_{2} y+\delta_{2} z+\int-\alpha_{2}{ }^{2} \phi_{2}(t)+\beta_{2}{ }^{2} \phi_{3}(t) / \alpha_{2} d t\right)^{2}+e^{\delta_{3} z}+e^{\delta_{4} z}+e^{\delta_{3} z+\delta_{4} z}\right)^{2}} .
\end{aligned}
$$

If $\tau_{1}^{2}+\tau_{2}^{2}+\exp \left(\tau_{3}\right)+\exp \left(\tau_{4}\right)+\exp \left(\tau_{3}+\tau_{4}\right)+\varepsilon_{5}(t)$ $\longrightarrow \infty$, the lump solutions $u \longrightarrow 0$ at any $t$. By selecting the parameters $\delta_{1}=1, \delta_{2}=2, \delta_{3}=1.5, \delta_{4}=3, \alpha_{2}=1, \beta_{2}=3$, $\phi_{2}(t)=\cos (t), \phi_{3}(t)=\sin (t), x=1, y=1$, then plots of equation (25) are plotted in Figure 1. And also, by selecting the parameters $\delta_{1}=1, \delta_{2}=2, \delta_{3}=1.5, \delta_{4}=3, \alpha_{2}=1, \beta_{2}$ $=3, \phi_{2}(t)=1 / 4 \cos (2 t), \phi_{3}(t)=1 / 4 \sin (1+2 t), x=1, y=1$, then plots of equation (25) are plotted in Figure 2.

Type II

$$
\begin{aligned}
\varepsilon_{1}(t) & =\int-2 \frac{\delta_{1} \phi_{4}(t)\left(\beta_{1} \delta_{2}-\beta_{2} \delta_{1}\right)}{\alpha_{2} \beta_{1}} d t, \varepsilon_{2}(t) \\
& =\int-\frac{\alpha_{2}^{2} \beta_{1}^{2} \phi_{2}(t)+\beta_{1}^{2} \delta_{2}^{2} \phi_{4}(t)-\beta_{2}^{2} \delta_{1}^{2} \phi_{4}(t)}{\beta_{1}^{2} \alpha_{2}} d t, \varepsilon_{4}(t)=\varepsilon_{5}(t)=0,
\end{aligned}
$$

$$
\begin{aligned}
\varepsilon_{3}(t) & =\int-2 \frac{\beta_{3} \delta_{1} \phi_{4}(t)\left(\beta_{1} \delta_{2}-\beta_{2} \delta_{1}\right)}{\beta_{1}{ }^{2} \alpha_{2}} d t, \alpha_{1} \\
& =\alpha_{3}=\alpha_{4}=0, \alpha_{2}=\alpha_{2}, \beta_{1}=\beta_{1}, \beta_{2}=\beta_{2}, \beta_{3}=\beta_{3}, \beta_{4}=0 \\
\delta_{1} & =\delta_{1}, \delta_{2}=\delta_{2}, \delta_{3}=\frac{\beta_{3} \delta_{1}}{\beta_{1}}, \delta_{4} \\
& =0, \phi_{1}(t)=0, \phi_{3}(t)=-\frac{\delta_{1}{ }^{2} \phi_{4}(t)}{\beta_{1}{ }^{2}} .
\end{aligned}
$$

The solutions are given as follows: 


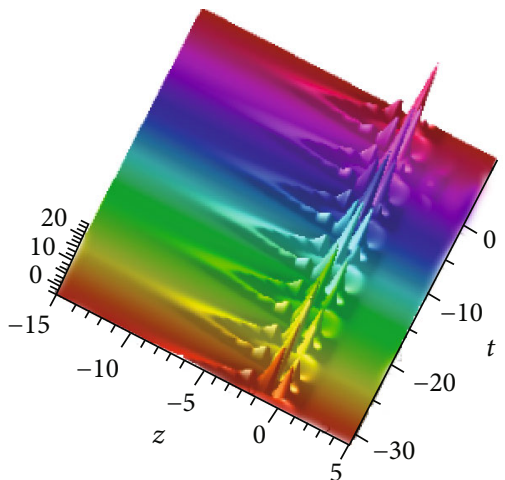

(a)

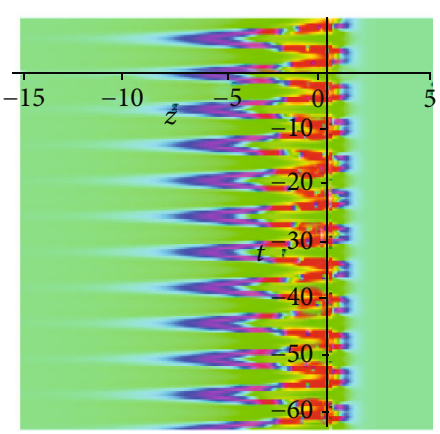

(b)

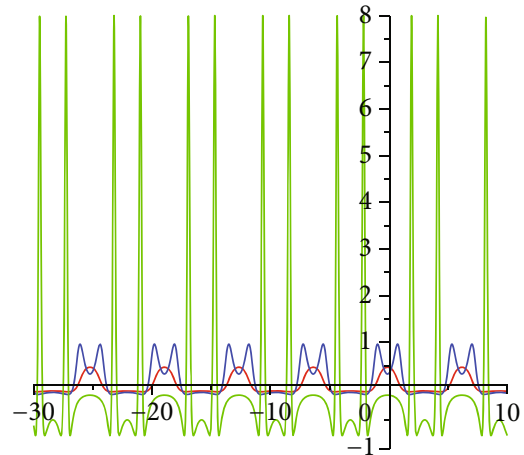

(c)

$-z=-7$

$-z=-5$

$-z=0$

Figure 1: Plots of interaction lump with two solitons (25).

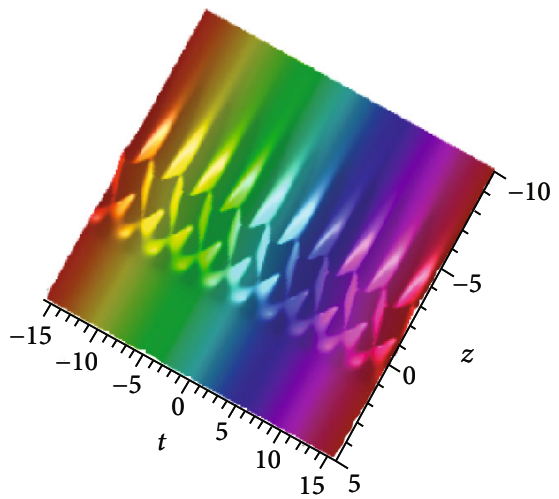

(a)

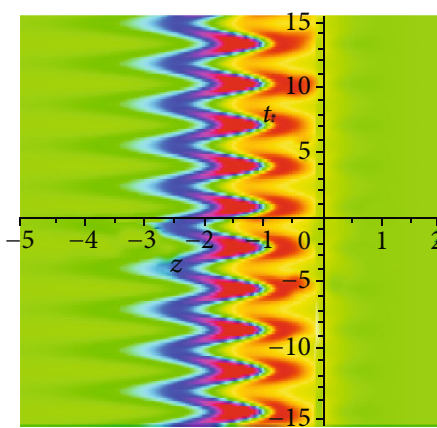

(b)
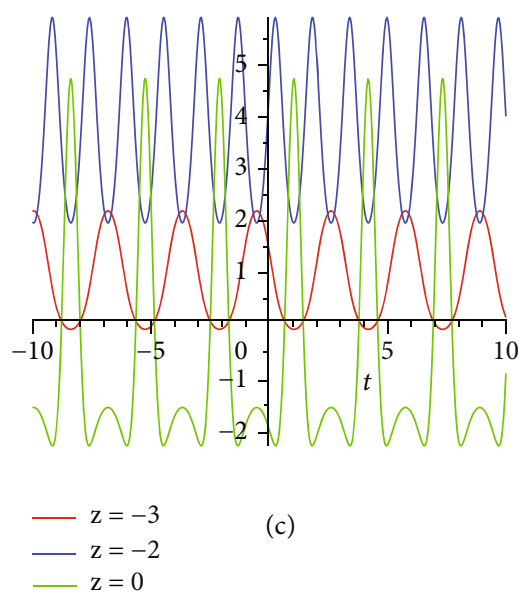

(c)

Figure 2: Plots of interaction lump with two solitons (25).

$$
\begin{aligned}
u_{2}= & \frac{24 \alpha_{2}^{2}}{\tau_{1}{ }^{2}+\tau_{2}{ }^{2}+2 e^{\beta_{3} y+\beta_{3} \delta_{1} z / \beta_{1}+\int-2 \beta_{3} \delta_{1} \phi_{4}(t)\left(\beta_{1} \delta_{2}-\beta_{2} \delta_{1}\right) / \beta_{1}{ }^{2} \alpha_{2} d t}} \\
& -\frac{48 \tau_{2}{ }^{2} \alpha_{2}{ }^{2}}{\left(\tau_{1}{ }^{2}+\tau_{2}{ }^{2}+2 e^{\beta_{3} y+\beta_{3} \delta_{1} z / \beta_{1}+\int-2 \beta_{3} \delta_{1} \phi_{4}(t)\left(\beta_{1} \delta_{2}-\beta_{2} \delta_{1}\right) / \beta_{1}{ }^{2} \alpha_{2} d t}\right)^{2}},
\end{aligned}
$$

$$
\begin{aligned}
\tau_{1} & =\beta_{1} y+\delta_{1} z+\int-2 \frac{\delta_{1} \phi_{4}(t)\left(\beta_{1} \delta_{2}-\beta_{2} \delta_{1}\right)}{\alpha_{2} \beta_{1}} d t, \tau_{2} \\
& =\alpha_{2} x+\beta_{2} y+\delta_{2} z+\int-\frac{\alpha_{2}{ }^{2} \beta_{1}{ }^{2} \phi_{2}(t)+\beta_{1}{ }^{2} \delta_{2}{ }^{2} \phi_{4}(t)-\beta_{2}{ }^{2} \delta_{1}{ }^{2} \phi_{4}(t)}{\beta_{1}{ }^{2} \alpha_{2}} d t .
\end{aligned}
$$

If $\tau_{1}^{2}+\tau_{2}^{2}+\exp \left(\tau_{3}\right)+\exp \left(\tau_{4}\right)+\exp \left(\tau_{3}+\tau_{4}\right)+\varepsilon_{5}(t)$ $\longrightarrow \infty$, the lump solutions $u \longrightarrow 0$ at any $t$. By selecting the parameters $\delta_{1}=1, \delta_{2}=2, \alpha_{2}=1, \beta_{1}=2, \beta_{2}=3, \beta_{3}=4$, $\phi_{2}(t)=\cos (t), \phi_{4}(t)=\sin (t), x=1, y=1$, then plots of equation (27) are plotted in Figure 3.
Type III

$$
\begin{aligned}
\varepsilon_{1}(t) & =\int 2 \frac{\delta_{1} \phi_{4}(t)\left(\beta_{2} \delta_{4}-\beta_{4} \delta_{2}\right)}{\alpha_{2} \beta_{4}} d t, \varepsilon_{2}(t) \\
& =\int-\frac{\alpha_{2}{ }^{2} \beta_{4}{ }^{2} \phi_{2}(t)-\beta_{2}{ }^{2} \delta_{4}{ }^{2} \phi_{4}(t)+\beta_{4}{ }^{2} \delta_{2}{ }^{2} \phi_{4}(t)}{\beta_{4}{ }^{2} \alpha_{2}} d t, \varepsilon_{5}(t)=0, \\
\varepsilon_{3}(t) & =\int 2 \frac{\delta_{3} \phi_{4}(t)\left(\beta_{2} \delta_{4}-\beta_{4} \delta_{2}\right)}{\alpha_{2} \beta_{4}} d t, \varepsilon_{4}(t) \\
& =\int 2 \frac{\delta_{4} \phi_{4}(t)\left(\beta_{2} \delta_{4}-\beta_{4} \delta_{2}\right)}{\alpha_{2} \beta_{4}} d t, \alpha_{1}=\alpha_{3}=\alpha_{4}=0, \alpha_{2}=\alpha_{2},
\end{aligned}
$$

$$
\begin{aligned}
\beta_{1} & =\frac{\beta_{4} \delta_{1}}{\delta_{4}}, \beta_{2}=\beta_{2}, \beta_{3}=\frac{\delta_{3} \beta_{4}}{\delta_{4}}, \beta_{4} \\
& =\beta_{4}, \delta_{1}=\delta_{1}, \delta_{2}=\delta_{2}, \delta_{3}=\delta_{3}, \delta_{4} \\
& =\delta_{4}, \phi_{1}(t)=0, \phi_{3}(t)=-\frac{\delta_{4}{ }^{2} \phi_{4}(t)}{\beta_{4}{ }^{2}} .
\end{aligned}
$$




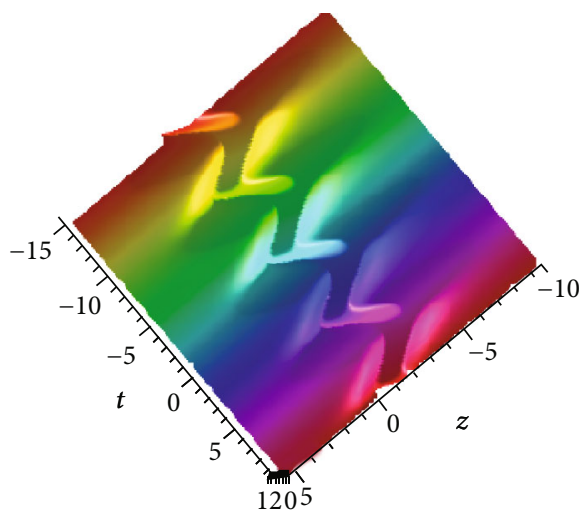

(a)

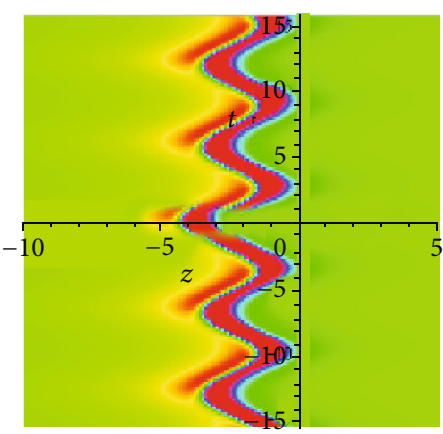

(b)

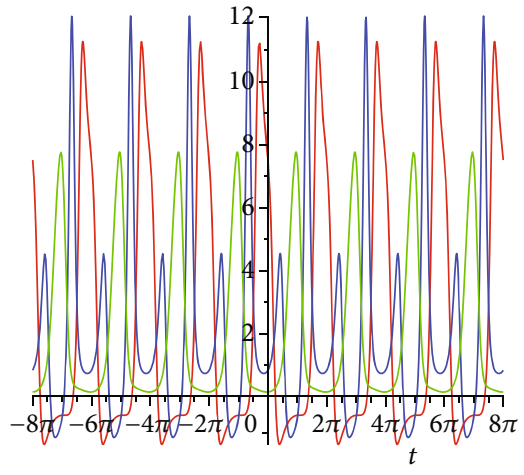

$-z=-3$

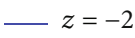

$z=-1$

(c)

FIgURE 3: Plots of interaction lump with two solitons (27).

The solutions are given as follows:

$u_{3}=24 \frac{\alpha_{2}{ }^{2}}{\tau_{1}{ }^{2}+\tau_{2}{ }^{2}+F_{1}+F_{2}+F_{3}}-48 \frac{\tau_{2}{ }^{2} \alpha_{2}{ }^{2}}{\left(\tau_{1}{ }^{2}+\tau_{2}{ }^{2}+F_{1}+F_{2}+F_{3}\right)^{2}}$,

$$
\begin{aligned}
\tau_{1} & =\frac{\beta_{4} \delta_{1} y}{\delta_{4}}+\delta_{1} z+\int 2 \frac{\delta_{1} \phi_{4}(t)\left(\beta_{2} \delta_{4}-\beta_{4} \delta_{2}\right)}{\alpha_{2} \beta_{4}} d t, \tau_{2} \\
& =\alpha_{2} x+\beta_{2} y+\delta_{2} z+\int-\frac{\alpha_{2}{ }^{2} \beta_{4}{ }^{2} \phi_{2}(t)-\beta_{2}{ }^{2} \delta_{4}{ }^{2} \phi_{4}(t)+\beta_{4}{ }^{2} \delta_{2}{ }^{2} \phi_{4}(t)}{\beta_{4}{ }^{2} \alpha_{2}} d t,
\end{aligned}
$$

$$
\begin{aligned}
F_{1} & =e^{\delta_{3} \beta_{4} y / \delta_{4}+\delta_{3} z+\int 2 \delta_{3} \phi_{4}(t)\left(\beta_{2} \delta_{4}-\beta_{4} \delta_{2}\right) / \alpha_{2} \beta_{4} d t}, F_{2} \\
& =e^{\beta_{4} y+\delta_{4} z+2 \delta_{4} t \phi_{4}(t)\left(\beta_{2} \delta_{4}-\beta_{4} \delta_{2}\right) / \alpha_{2} \beta_{4}},
\end{aligned}
$$

$F_{3}=e^{\delta_{3} \beta_{4} y / \delta_{4}+\delta_{3} z+\int 2 \delta_{3} \phi_{4}(t)\left(\beta_{2} \delta_{4}-\beta_{4} \delta_{2}\right) / \alpha_{2} \beta_{4} d t+\beta_{4} y+\delta_{4} z+2 \delta_{4} t \phi_{4}(t)\left(\beta_{2} \delta_{4}-\beta_{4} \delta_{2}\right) / \alpha_{2} \beta_{4}}$.
By selecting the parameters $\delta_{1}=1, \delta_{2}=2, \delta_{3}=3, \delta_{4}=4$, $\alpha_{2}=1, \beta_{2}=3, \beta_{4}=4, \phi_{2}(t)=\cos (t), \phi_{4}(t)=\sin (t), x=1, y$ $=1$, then plots of equation (30) are plotted in Figure 4 .

Type IV

$$
\begin{aligned}
\varepsilon_{1}(t) & =-\frac{\varepsilon_{2}(t) \delta_{2}}{\delta_{1}}, \varepsilon_{3}(t) \\
& =\int-\frac{\alpha_{3}^{4} \phi_{1}(t)+\alpha_{3}{ }^{2} \phi_{2}(t)+\beta_{3}{ }^{2} \phi_{3}(t)}{\alpha_{3}} d t, \varepsilon_{4}(t) \\
& =0, \varepsilon_{5}(t)=-2 \frac{\varepsilon_{2}(t)\left(\delta_{1} \varepsilon_{2}(t)-\delta_{2} \varepsilon_{1}(t)\right)}{\delta_{1}}, \\
\alpha_{1} & =\alpha_{2}=\alpha_{4}=0, \alpha_{3}=\alpha_{3}, \beta_{1}=\beta_{2}=\beta_{4}=0, \beta_{3} \\
& =\beta_{3}, \delta_{1}=\delta_{1}, \delta_{2}=\delta_{2}, \delta_{3}=\delta_{3}, \delta_{4}=\delta_{4}, \phi_{4}(t)=0 .
\end{aligned}
$$

The solutions are given as follows:

$$
\begin{gathered}
u_{4}=\frac{12 \alpha_{3}{ }^{2} F_{1}+12 \alpha_{3}{ }^{2} F_{2}}{\left(\delta_{1} z-\varepsilon_{2}(t) \delta_{2} / \delta_{1}\right)^{2}+\left(\delta_{2} z+\varepsilon_{2}(t)\right)^{2}+F_{1}+e^{\delta_{4} z}+F_{2}-2 \varepsilon_{2}(t)\left(\delta_{1} \varepsilon_{2}(t)-\delta_{2} \varepsilon_{1}(t)\right) / \delta_{1}}, \\
\frac{12\left(\alpha_{3} F_{1}+\alpha_{3} F_{2}\right)^{2}}{\left(\left(\delta_{1} z-\varepsilon_{2}(t) \delta_{2} / \delta_{1}\right)^{2}+\left(\delta_{2} z+\varepsilon_{2}(t)\right)^{2}+F_{1}+e^{\delta_{4} z}+F_{2}-2 \varepsilon_{2}(t)\left(\delta_{1} \varepsilon_{2}(t)-\delta_{2} \varepsilon_{1}(t)\right) / \delta_{1}\right)^{2}}, \\
F_{1}=e^{\alpha_{3} x+\beta_{3} y+\delta_{3} z+\int-\alpha_{3}{ }^{4} \phi_{1}(t)+\alpha_{3}{ }^{2} \phi_{2}(t)+\beta_{3}{ }^{2} \phi_{3}(t) / \alpha_{3} d t}, F_{2}=e^{\alpha_{3} x+\beta_{3} y+\delta_{3} z+\int-\alpha_{3}{ }^{4} \phi_{1}(t)+\alpha_{3}{ }^{2} \phi_{2}(t)+\beta_{3}{ }^{2} \phi_{3}(t) / \alpha_{3} d t+\delta_{4} z} .
\end{gathered}
$$

By selecting the parameters $\delta_{1}=1, \delta_{2}=2, \delta_{3}=3, \delta_{4}=4$, $\alpha_{2}=1, \beta_{2}=3, \beta_{4}=4, \phi_{2}(t)=\cos (t), \phi_{4}(t)=\sin (t), x=1, y$ $=1$, then plots of equation (35) are plotted in Figure 5 .

Type $V$

$$
\varepsilon_{1}(t)=-\frac{\varepsilon_{2}(t) \delta_{2}}{\delta_{1}}, \varepsilon_{3}(t)
$$

$$
\begin{aligned}
& =\int-\alpha_{3}\left(\alpha_{3}^{2} \phi_{1}(t)+\phi_{2}(t)\right) d t, \varepsilon_{4}(t)=0, \varepsilon_{5}(t) \\
& =\int-2 \frac{\left(d / d t \varepsilon_{2}(t)\right)\left(\delta_{1} \varepsilon_{2}(t)-\delta_{2} \varepsilon_{1}(t)\right)}{\delta_{1}} d t, \\
\alpha_{1} & =\alpha_{2}=\alpha_{4}=0, \alpha_{3}=\alpha_{3}, \beta_{1}=\beta_{2}=\beta_{4}=0, \beta_{3} \\
& =\beta_{3}, \delta_{1}=\delta_{1}, \delta_{2}=\delta_{2}, \delta_{3}=\delta_{3}, \delta_{4}=\delta_{4}, \phi_{3}(t)=\phi_{4}(t)=0 .
\end{aligned}
$$




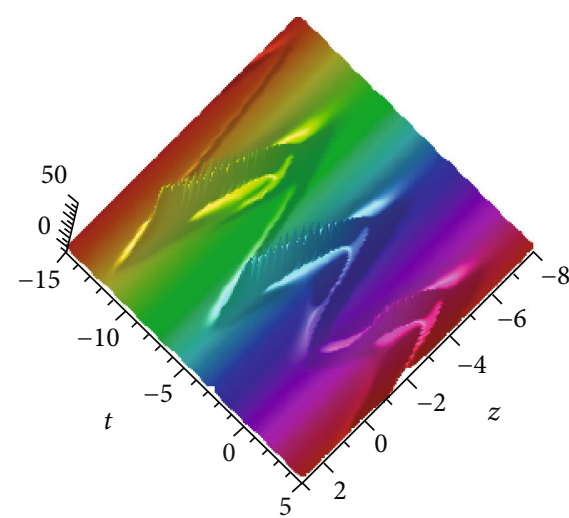

(a)

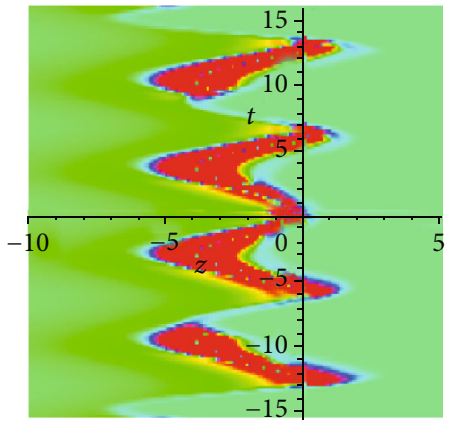

(b)

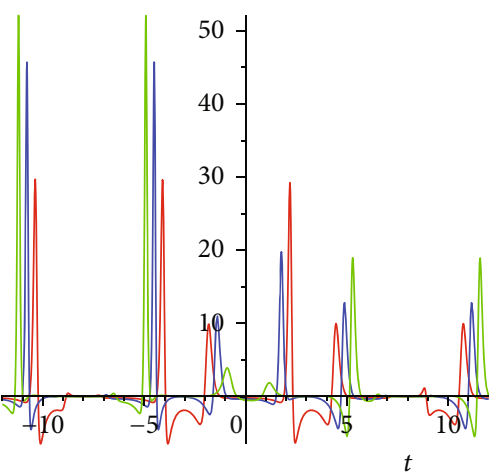

(c)

Figure 4: Plots of interaction lump with two solitons (30).

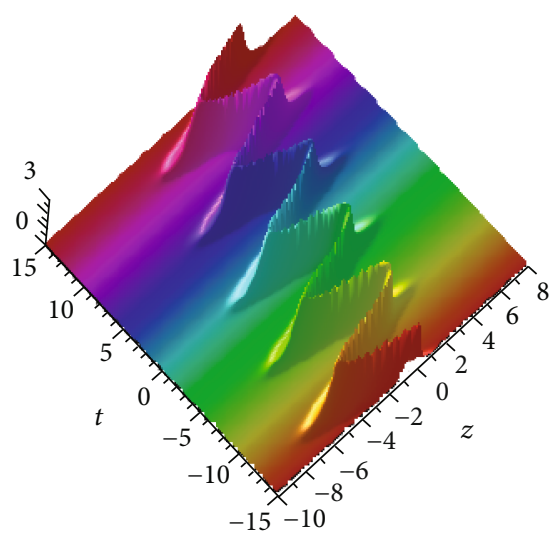

(a)

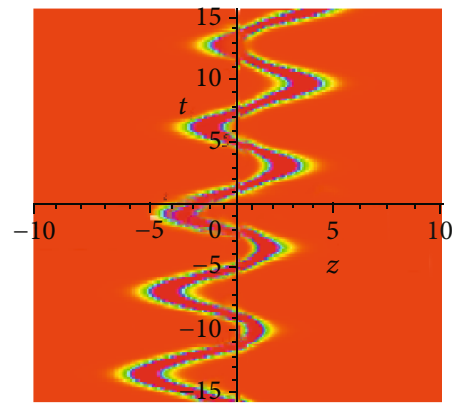

(b)

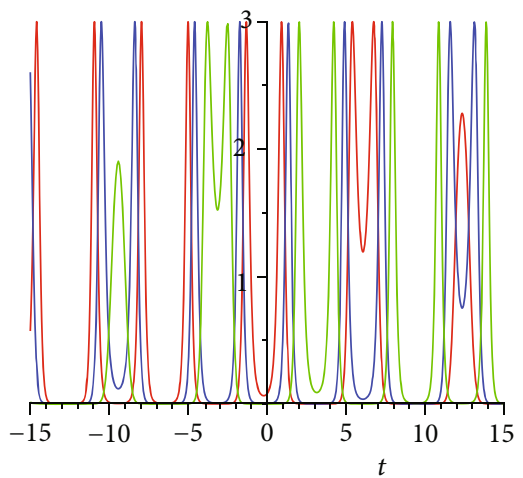

(c)

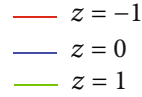

Figure 5: Plots of interaction lump with two solitons (35).

The solutions are given as follows:

$$
\begin{aligned}
u_{5}= & 12 \frac{\partial^{2}}{\partial x^{2}} \ln \left(\mathbf{F}_{5}\right) \\
\mathbf{F}_{5}= & \left(\delta_{1} z+\varepsilon_{1}(t)\right)^{2}+\left(\delta_{2} z+\varepsilon_{2}(t)\right)^{2}+e^{\alpha_{3} x+\beta_{3} y+\delta_{3} z+\varepsilon_{3}(t)} \\
& +e^{\beta_{4} y+\delta_{4} z+\varepsilon_{4}(t)}+e^{\alpha_{3} x+\beta_{3} y+\delta_{3} z+\varepsilon_{3}(t)+\beta_{4} y+\delta_{4} z+\varepsilon_{4}(t)}+\varepsilon_{5}(t)
\end{aligned}
$$

\section{Type VI}

$$
\begin{aligned}
\varepsilon_{3}(t) & =\int-\frac{\alpha_{3}{ }^{4} \phi_{1}(t)+\alpha_{3}{ }^{2} \phi_{2}(t)+\delta_{3}{ }^{2} \phi_{3}(t)}{\alpha_{3}} d t, \varepsilon_{2}(t) \\
& =\varepsilon_{4}(t)=0, \varepsilon_{5}(t)=-\left(\varepsilon_{1}(t)\right)^{2}, \\
\alpha_{1} & =\alpha_{2}=\alpha_{4}=0, \alpha_{3}=\alpha_{3}, \beta_{1}=0, \beta_{2}=\beta_{2}, \beta_{3} \\
& =\beta_{3}, \beta_{4}=\beta_{4}, \delta_{1}=\delta_{2}=\delta_{4}=0, \delta_{3}=\delta_{3}, \phi_{3}(t)=0 .
\end{aligned}
$$

The solutions are given as follows:

$$
\begin{aligned}
u_{5}= & 12 \frac{\partial^{2}}{\partial x^{2}} \ln \left(\mathbf{F}_{6}\right), \mathbf{F}_{6}=\left(\beta_{2} y+\varepsilon_{2}(t)\right)^{2}+e^{\alpha_{3} x+\beta_{3} y+\delta_{3} z+\varepsilon_{3}(t)} \\
& +e^{\beta_{4} y+\varepsilon_{4}(t)}+e^{\alpha_{3} x+\beta_{3} y+\delta_{3} z+\varepsilon_{3}(t)+\beta_{4} y+\varepsilon_{4}(t)}
\end{aligned}
$$

Type VII

$$
\begin{aligned}
\varepsilon_{3}(t) & =\int-\alpha_{3}\left(\alpha_{3}^{2} \phi_{1}(t)+\phi_{2}(t)\right) d t, \varepsilon_{1}(t)=\varepsilon_{2}(t) \\
& =\varepsilon_{4}(t)=\varepsilon_{5}(t)=0, \alpha_{1}=\alpha_{2}=\alpha_{4}=0, \alpha_{3}=\alpha_{3}, \\
\beta_{1} & =0, \beta_{2}=\beta_{2}, \beta_{3}=\beta_{3}, \beta_{4}=\beta_{4}, \delta_{1}=\delta_{1}, \delta_{2} \\
& =\delta_{2}, \delta_{3}=\delta_{3}, \delta_{4}=\delta_{4}, \phi_{3}(t)=\phi_{4}(t)=0 .
\end{aligned}
$$

The solutions are given as follows: 


$$
\begin{aligned}
u_{7}= & 12 \frac{\partial^{2}}{\partial x^{2}} \ln \left(\mathbf{F}_{7}\right) \\
\mathbf{F}_{7}= & z^{2} \delta_{1}{ }^{2}+\left(\beta_{2} y+\delta_{2} z\right)^{2}+e^{\alpha_{3} x+\beta_{3} y+\delta_{3} z+\int-\alpha_{3}\left(\alpha_{3}{ }^{2} \phi_{1}(t)+\phi_{2}(t)\right) d t} \\
& +e^{\beta_{4} y+\delta_{4} z}+e^{\alpha_{3} x+\beta_{3} y+\delta_{3} z+\int-\alpha_{3}\left(\alpha_{3}{ }^{2} \phi_{1}(t)+\phi_{2}(t)\right) d t+\beta_{4} y+\delta_{4} z}
\end{aligned}
$$

Type VIII

$$
\begin{aligned}
\varepsilon_{2}(t) & =\int-2 \frac{\delta_{2} \phi_{4}(t)\left(\beta_{2} \delta_{3}-\beta_{3} \delta_{2}\right)}{\alpha_{3} \beta_{2}} d t, \varepsilon_{3}(t) \\
& =\int-\frac{\alpha_{3}{ }^{4} \beta_{2}{ }^{2} \phi_{1}(t)+\alpha_{3}{ }^{2} \beta_{2}{ }^{2} \phi_{2}(t)+\beta_{2}{ }^{2} \delta_{3}{ }^{2} \phi_{4}(t)-\beta_{3}{ }^{2} \delta_{2}{ }^{2} \phi_{4}(t)}{\beta_{2}{ }^{2} \alpha_{3}} d t \\
\varepsilon_{4}(t) & =\int-2 \frac{\left(\beta_{2} \delta_{3}-\beta_{3} \delta_{2}\right) \delta_{4} \phi_{4}(t)}{\alpha_{3} \beta_{2}} d t, \varepsilon_{5}(t) \\
& =-\varepsilon_{1}^{2}(t), \alpha_{1}=\alpha_{2}=\alpha_{4}=0, \alpha_{3}=\alpha_{3}, \\
\beta_{1} & =0, \beta_{2}=\beta_{2}, \beta_{3}=\beta_{3}, \beta_{4}=\frac{\beta_{2} \delta_{4}}{\delta_{2}}, \delta_{1}=\delta_{1}, \delta_{2} \\
& =\delta_{2}, \delta_{3}=\delta_{3}, \delta_{4}=\delta_{4}, \phi_{3}(t)=-\frac{\delta_{2}{ }^{2} \phi_{4}(t)}{\beta_{2}{ }^{2}} .
\end{aligned}
$$

The solutions are given as follows:

$u_{8}=12 \frac{\partial^{2}}{\partial x^{2}} \ln \left(\mathbf{F}_{8}\right)$

$$
\begin{aligned}
\mathbf{F}_{8}= & \left(\varepsilon_{1}(t)\right)^{2}+\left(\beta_{2} y+\delta_{2} z+\varepsilon_{2}(t)\right)^{2}+e^{\alpha_{3} x+\beta_{3} y+\delta_{3} z+\varepsilon_{3}(t)} \\
& +e^{\beta_{2} \delta_{4} y / \delta_{2}+\delta_{4} z+\varepsilon_{4}(t)}+e^{\alpha_{3} x+\beta_{3} y+\delta_{3} z+\varepsilon_{3}(t)+\beta_{2} \delta_{4} y / \delta_{2}+\delta_{4} z+\varepsilon_{4}(t)}+\varepsilon_{5}(t) .
\end{aligned}
$$

Type IX

$$
\begin{gathered}
\varepsilon_{1}(t)=-\frac{\varepsilon_{2}(t) \beta_{2}}{\beta_{1}}, \varepsilon_{3}(t)=\int-\alpha_{3}\left(\alpha_{3}^{2} \phi_{1}(t)+\phi_{2}(t)\right) d t, \varepsilon_{4}(t) \\
=0, \varepsilon_{5}(t)=\int-2 \frac{\left(d / d t \varepsilon_{2}(t)\right)\left(\beta_{1} \varepsilon_{2}(t)-\beta_{2} \varepsilon_{1}(t)\right)}{\beta_{1}} d t, \\
\alpha_{1}=\alpha_{2}=\alpha_{4}=0, \alpha_{3}=\alpha_{3}, \beta_{1}=\beta_{1}, \beta_{2}=\beta_{2}, \beta_{3}=\beta_{3}, \beta_{4}=\beta_{4}, \\
\delta_{1}=\delta_{1}, \delta_{2}=\frac{\beta_{2} \delta_{1}}{\beta_{1}}, \delta_{3}=\delta_{3}, \delta_{4}=\delta_{4}, \phi_{3}(t)=\phi_{4}(t)=0 .
\end{gathered}
$$

The solutions are given as follows:

$$
\begin{aligned}
u_{9}= & 12 \frac{\partial^{2}}{\partial x^{2}} \ln \left(\mathbf{F}_{9}\right), \mathbf{F}_{9}=\left(\beta_{1} y+\delta_{1} z+\varepsilon_{1}(t)\right)^{2} \\
& +\left(\beta_{2} y+\frac{\beta_{2} \delta_{1} z}{\beta_{1}}+\varepsilon_{2}(t)\right)^{2}+e^{\alpha_{3} x+\beta_{3} y+\delta_{3} z+\varepsilon_{3}(t)}+e^{\beta_{4} y+\delta_{4} z+\varepsilon_{4}(t)} \\
& +e^{\alpha_{3} x+\beta_{3} y+\delta_{3} z+\varepsilon_{3}(t)+\beta_{4} y+\delta_{4} z+\varepsilon_{4}(t)}+\varepsilon_{5}(t) .
\end{aligned}
$$

Type X

$$
\begin{aligned}
\varepsilon_{1}(t) & =\int-\frac{2 \beta_{1}{ }^{3} \delta_{1} \delta_{3} \phi_{4}(t)-2 \beta_{1}{ }^{2} \beta_{3} \delta_{1}{ }^{2} \phi_{4}(t)+2 \beta_{1} \beta_{2}{ }^{2} \delta_{1} \delta_{3} \phi_{4}(t)-2 \beta_{2}{ }^{2} \beta_{3} \delta_{1}{ }^{2} \phi_{4}(t)+\left(d / d t \varepsilon_{2}(t)\right) \alpha_{3} \beta_{1}{ }^{2} \beta_{2}}{\alpha_{3} \beta_{1}{ }^{3}} d t, \\
\varepsilon_{3}(t) & =\int-\frac{\alpha_{3}{ }^{4} \beta_{1}{ }^{2} \phi_{1}(t)+\alpha_{3}{ }^{2} \beta_{1}{ }^{2} \phi_{2}(t)+\beta_{1}{ }^{2} \delta_{3}{ }^{2} \phi_{4}(t)-\beta_{3}{ }^{2} \delta_{1}{ }^{2} \phi_{4}(t)}{\alpha_{3} \beta_{1}{ }^{2}} d t, \varepsilon_{4}(t)=\int-2 \frac{\left(\beta_{1} \delta_{3}-\beta_{3} \delta_{1}\right) \delta_{4} \phi_{4}(t)}{\alpha_{3} \beta_{1}} d t \\
\varepsilon_{5}(t) & =\int-2 \frac{\left(\varepsilon_{2}(t) \beta_{1}{ }^{4} \alpha_{3}+\int S_{1}+\left(d / d t \varepsilon_{2}(t)\right) \alpha_{3} \beta_{1}{ }^{2} \beta_{2} d t \beta_{2}\right)\left(S_{2}+\left(d / d t \varepsilon_{2}(t)\right) \alpha_{3} \beta_{1}{ }^{2}\right)}{\alpha_{3}{ }^{2} \beta_{1}{ }^{6}} d t \\
S_{1} & =2 \delta_{1} \phi_{4}(t)\left(\beta_{1}{ }^{2}+\beta_{2}{ }^{2}\right)\left(\beta_{1} \delta_{3}-\beta_{3} \delta_{1}\right), S_{2}=2 \beta_{2} \delta_{1} \phi_{4}(t)\left(\beta_{1} \delta_{3}-\beta_{3} \delta_{1}\right), \\
\alpha_{1} & =\alpha_{2}=\alpha_{4}=0, \beta_{4}=\frac{\beta_{1} \delta_{4}}{\delta_{1}}, \delta_{1}=\delta_{1}, \delta_{2}=\frac{\beta_{2} \delta_{1}}{\beta_{1}}, \delta_{3}=\delta_{3}, \delta_{4}=\delta_{4}, \phi_{3}(t)=-\frac{\delta_{1}{ }^{2} \phi_{4}(t)}{\beta_{1}{ }^{2}} .
\end{aligned}
$$

The solutions are given as follows:

$$
\begin{aligned}
u_{10}= & 12 \frac{\partial^{2}}{\partial x^{2}} \ln \left(\mathbf{F}_{10}\right), \mathbf{F}_{10}=\left(\beta_{1} y+\delta_{1} z+\varepsilon_{1}(t)\right)^{2} \\
& +\left(\beta_{2} y+\frac{\beta_{2} \delta_{1} z}{\beta_{1}}+\varepsilon_{2}(t)\right)^{2}+e^{\alpha_{3} x+\beta_{3} y+\delta_{3} z+\varepsilon_{3}(t)} \\
& +e^{\beta_{1} \delta_{4} y / \delta_{1}+\delta_{4} z+\varepsilon_{4}(t)}+e^{\alpha_{3} x+\beta_{3} y+\delta_{3} z+\varepsilon_{3}(t)+\beta_{1} \delta_{4} y / \delta_{1}+\delta_{4} z+\varepsilon_{4}(t)}+\varepsilon_{5}(t) .
\end{aligned}
$$

By selecting the parameters $\delta_{1}=1, \delta_{3}=3, \delta_{4}=4, \alpha_{3}=1$, $\beta_{1}=4, \beta_{2}=2, \beta_{3}=3, \phi_{1}(t)=\sin (2 t), \phi_{2}(t)=\cos (2 t), \phi_{4}(t)$ $=\cos (3 t), \varepsilon_{2}(t)=\exp (t), x=1, y=1$, then plots of equation (49) are plotted in Figure 6. And also, by choosing the parameters $\delta_{1}=1, \delta_{3}=3, \delta_{4}=4, \alpha_{3}=1, \beta_{1}=4, \beta_{2}=2, \beta_{3}=$ $3, \phi_{1}(t)=\exp (t) \sin (t), \phi_{2}(t)=\exp (t) \cos (2 t), \phi_{4}(t)=\exp$ $(t) \cos (3 t), \varepsilon_{2}(t)=\exp (2 t), x=1, y=1$, then plots of equation (49) are plotted in Figure 7. Moreover, by choosing the parameters $\delta_{1}=1, \delta_{3}=3, \delta_{4}=4, \alpha_{3}=1, \beta_{1}=4, \beta_{2}=2, \beta_{3}$ $=3, \phi_{1}(t)=1 / 1+\sin (t), \phi_{2}(t)=\cos (t) /(2+\sin (t)), \phi_{4}(t)$ $=\cos (t), \varepsilon_{2}(t)=\sin ^{2}(t)+\tan (t), x=1, y=1$, then plots of equation (49) are plotted in Figure 8. 


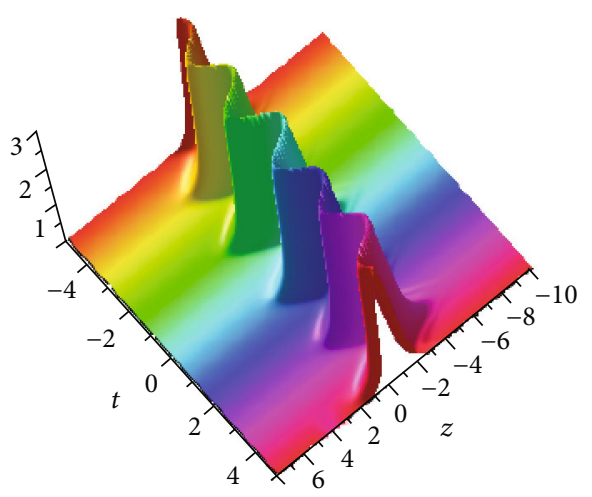

(a)

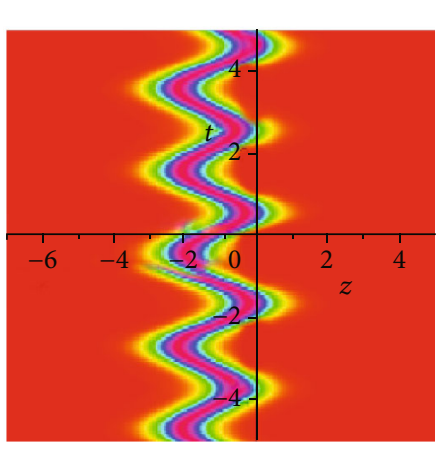

(b)

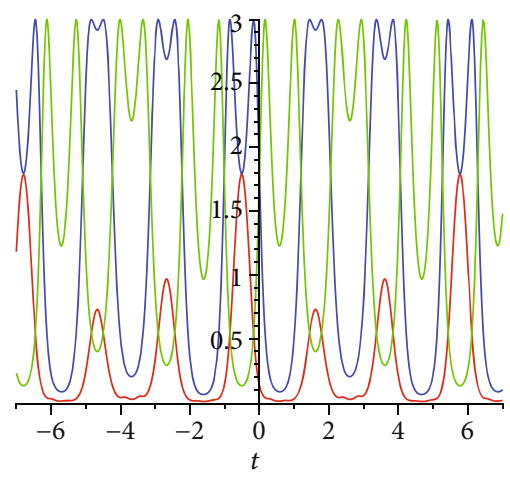

$-z=-3$

$-z=-2$

Figure 6: Plots of interaction lump with two solitons (49).

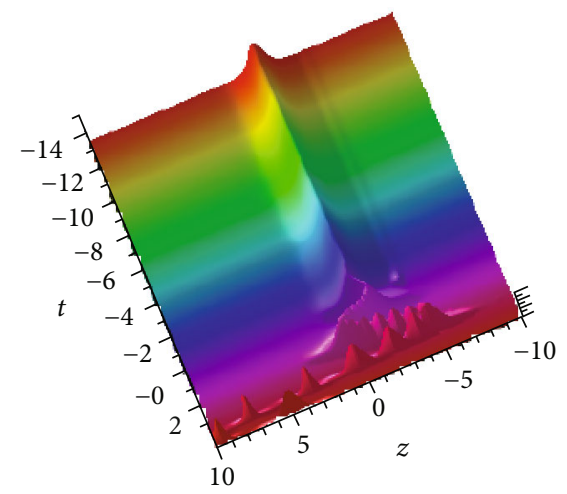

(a)

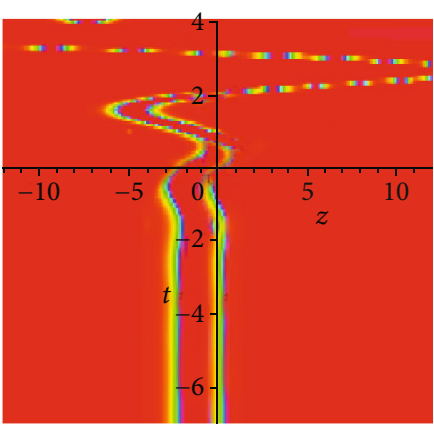

(b)

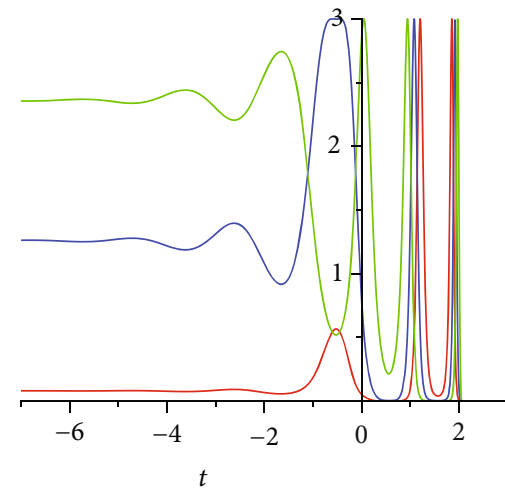

(c)

Figure 7: Plots of interaction lump with two solitons (49).

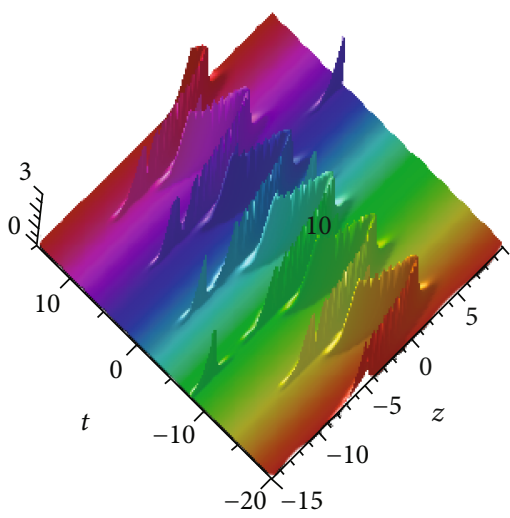

(a)

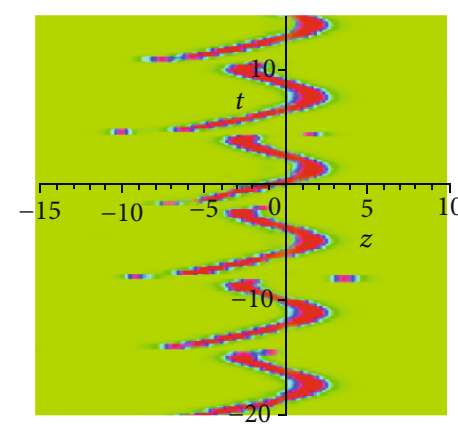

(b)

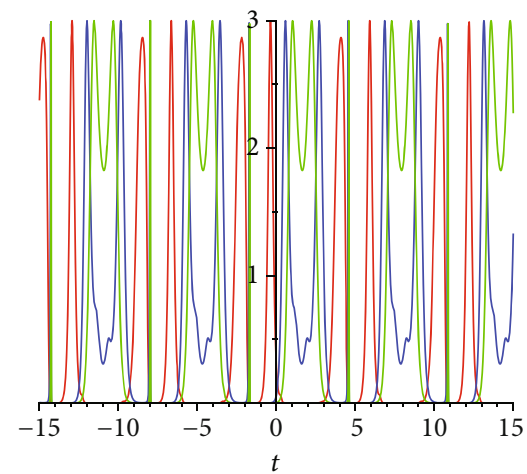

(c)

FIGURE 8: Plots of interaction lump with two solitons (49). 
4.2. Interaction between Two Lump Solutions. Here, we offer interaction between two lump solutions containing combination of two functions for the $(3+1)$-dimensional variable-coefficient nonlinear wave equation through utilizing the bilinear method as the below frame:

$g=\tau_{1}^{4}+\tau_{2}^{2}+\tau_{3}^{2}+\varepsilon_{4}(t), \tau_{j}=\alpha_{j} x+\beta_{j} y+\delta_{j} z+\varepsilon_{j}(t), j=1,2,3, \varepsilon_{4}(t)>0$.

The values $\alpha_{i}, \beta_{i}, \delta_{i}, \varepsilon_{i}(t)(i=1,2,3)$ are real constants to be computed. By appending (50) into (22), we receive to a system containing 42 nonlinear equations. To solve the nonlinear system, the determined coefficients will be got as the below cases.

Type I

$$
\begin{aligned}
\varepsilon_{1}(t)= & \sqrt[4]{C_{1}-\varepsilon_{4}(t)}, \varepsilon_{2}(t) \\
= & \int-2 \frac{\beta_{2} \beta_{3} \phi_{3}(t)+\delta_{2} \delta_{3} \phi_{4}(t)}{\alpha_{3}} d t+C_{2}, \alpha_{1}=\alpha_{2}=0, \alpha_{3}=\alpha_{3}, \\
\varepsilon_{3}(t)= & \int-\frac{\alpha_{3}{ }^{2} \phi_{2}(t)-\phi_{3}(t) \beta_{2}{ }^{2}+\beta_{3}{ }^{2} \phi_{3}(t)-\delta_{2}{ }^{2} \phi_{4}(t)+\delta_{3}{ }^{2} \phi_{4}(t)}{\alpha_{3}} d t \\
& +C_{3}, \beta_{1}=0, \beta_{2}=\beta_{2},
\end{aligned}
$$

$$
\beta_{3}=\beta_{3}, \delta_{1}=0, \phi_{1}(t)=-\frac{1}{3} \frac{\left(r_{1}^{4}+r_{4}\right)\left(\delta_{2}^{2} \phi_{4}(t)+\phi_{3}(t) \beta_{2}^{2}\right)}{\alpha_{3}{ }^{4}} .
$$

The solutions are given as follows:

$$
\begin{aligned}
u_{1}= & 12 \frac{\partial^{2}}{\partial x^{2}} \ln \left(\mathbf{F}_{1}\right), \mathbf{F}_{1}=\left(\varepsilon_{1}(t)\right)^{4}+\left(\beta_{2} y+\delta_{2} z+\varepsilon_{2}(t)\right)^{2} \\
& +\left(\alpha_{3} x+\beta_{3} y+\delta_{3} z+\varepsilon_{3}(t)\right)^{2}+\varepsilon_{4}(t) .
\end{aligned}
$$

If $\tau_{1}^{4}+\tau_{2}^{2}+\tau_{3}^{2}+\varepsilon_{4}(t) \longrightarrow \infty$, the lump solutions $u \longrightarrow 0$ at any $t$. By selecting the parameters $\delta_{2}=2, \delta_{3}=3, \alpha_{3}=3, \beta_{2}$ $=2, \beta_{3}=1, \phi_{2}(t)=t, \phi_{3}(t)=t^{2}, \phi_{4}(t)=1+t, x=1, z=1$,

then plots of equation (52) are plotted in Figure 9. And also, by selecting the parameters $\delta_{2}=2, \delta_{3}=3, \alpha_{3}=3, \beta_{2}=2, \beta_{3}=1$, $\phi_{2}(t)=\cos (t), \phi_{3}(t)=\sin (t), \phi_{4}(t)=\cos (2 t), x=1, z=1$, then plots of equation (52) are plotted in Figure 10. Moreover, by selecting the parameters $\delta_{2}=2, \delta_{3}=3, \alpha_{3}=3, \beta_{2}=2, \beta_{3}=$ $1, \phi_{2}(t)=t^{2} \sin (2 t), \phi_{3}(t)=t \sin (3 t), \phi_{4}(t)=t \cos (3 t), x=$ $1, z=1$, then plots of equation (52) are plotted in Figure 11.

Type II

$$
\begin{aligned}
& \varepsilon_{1}(t)=\frac{\sqrt[4]{-\left(-C_{1} \alpha_{2}{ }^{3}+\int F(t) d t\right) \alpha_{2}}}{\alpha_{2}}, \alpha_{1}=\beta_{1}=\delta_{1}=\phi_{1}(t)=0, \delta_{3}=\frac{\alpha_{3} \delta_{2}}{\alpha_{2}}, \\
& F(t)=2\left(\phi_{2}(t) \alpha_{2}{ }^{2} \alpha_{3}+\phi_{3}(t) \alpha_{3} \beta_{2}{ }^{2}+\phi_{4}(t) \alpha_{3} \delta_{2}{ }^{2}+\left(\frac{d}{d t} \varepsilon_{3}(t)\right) \alpha_{2}{ }^{2}\right)\left(\varepsilon_{3}(t) \alpha_{2}-\varepsilon_{2}(t) \alpha_{3}\right)+\left(\frac{d}{d t} \varepsilon_{4}(t)\right) \alpha_{2}{ }^{3} \\
& \varepsilon_{2}(t)=\int-\frac{\left(\alpha_{2}{ }^{2}+\alpha_{3}{ }^{2}\right)\left(\delta_{2}{ }^{2} \phi_{4}(t)+\phi_{3}(t) \beta_{2}{ }^{2}+\phi_{2}(t) \alpha_{2}{ }^{2}\right)+\left(d / d t \varepsilon_{3}(t)\right) \alpha_{2}{ }^{2} \alpha_{3}}{\alpha_{2}{ }^{3}} d t+C_{2} .
\end{aligned}
$$

The solutions are given as follows:

$$
\begin{aligned}
u_{2}= & 12 \frac{\partial^{2}}{\partial x^{2}} \ln \left(\mathbf{F}_{2}\right), \mathbf{F}_{2}=\left(\varepsilon_{1}(t)\right)^{4}+\left(\alpha_{2} x+\beta_{2} y+\delta_{2} z+\varepsilon_{2}(t)\right)^{2} \\
& +\left(\alpha_{3} x+\frac{\alpha_{3} \beta_{2} y}{\alpha_{2}}+\frac{\alpha_{3} \delta_{2} z}{\alpha_{2}}+\varepsilon_{3}(t)\right)^{2}+\varepsilon_{4}(t) .
\end{aligned}
$$

Type III

$$
\begin{aligned}
\varepsilon_{1}(t) & =\int-2 \frac{\phi_{4}(t) \delta_{1}\left(\beta_{1} \delta_{3}-\beta_{3} \delta_{1}\right)}{\alpha_{3} \beta_{1}} d t, \varepsilon_{3}(t) \\
& =\int-\frac{\alpha_{3}{ }^{2} \beta_{1}{ }^{2} \phi_{2}(t)+\beta_{1}{ }^{2} \delta_{3}{ }^{2} \phi_{4}(t)-\beta_{3}{ }^{2} \delta_{1}{ }^{2} \phi_{4}(t)}{\alpha_{3} \beta_{1}{ }^{2}} d t \\
\varepsilon_{4}(t) & =-\left(\varepsilon_{2}(t)\right)^{2}+C_{1}, \alpha_{1}=\alpha_{2}=\beta_{2} \\
& =\delta_{2}=\phi_{1}(t)=0, \phi_{3}(t)=-\frac{\delta_{1}{ }^{2} \phi_{4}(t)}{\beta_{1}{ }^{2}}
\end{aligned}
$$

The solutions are given as follows:

$$
\begin{aligned}
u_{3}= & 12 \frac{\partial^{2}}{\partial x^{2}} \ln \left(\mathbf{F}_{3}\right), \mathbf{F}_{3}=\left(\beta_{1} y+\delta_{1} z+\varepsilon_{1}(t)\right)^{4}+\left(\varepsilon_{2}(t)\right)^{2} \\
& +\left(\alpha_{3} x+\beta_{3} y+\delta_{3} z+\varepsilon_{3}(t)\right)^{2}+\varepsilon_{4}(t) .
\end{aligned}
$$

4.3. Interaction between Two Lumps-Soliton Solutions. Here, we offer interaction between two lumps-soliton solutions containing combination of two functions for the $(3+1)$-dimensional variable-coefficient nonlinear wave equation through utilizing the bilinear method as the below frame:

$$
\begin{aligned}
g & =\tau_{1}^{4}+\tau_{2}^{2}+\tau_{3}^{2}+\exp \left(\tau_{4}\right)+\varepsilon_{5}(t), \tau_{j} \\
& =\alpha_{j} x+\beta_{j} y+\delta_{j} z+\varepsilon_{j}(t), j \\
& =1,2,3,4, \varepsilon_{4}(t)>0 .
\end{aligned}
$$

The values $\alpha_{i}, \beta_{i}, \delta_{i}, \varepsilon_{i}(t)(i=1,2,3,4)$ are real constants to be computed. By putting (57) into (22), we receive a 


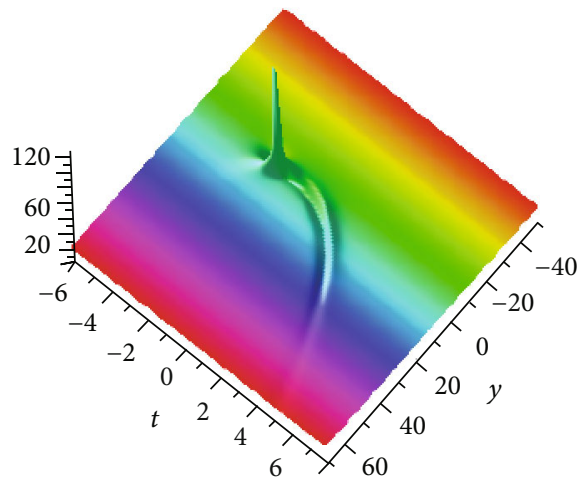

(a)

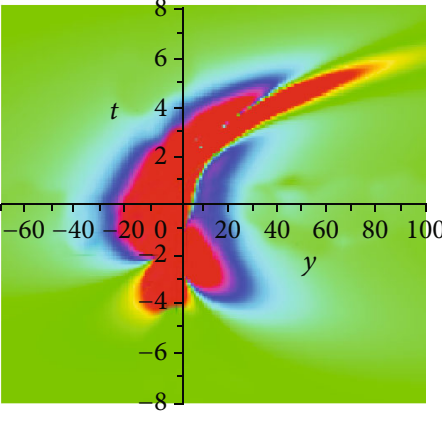

(b)

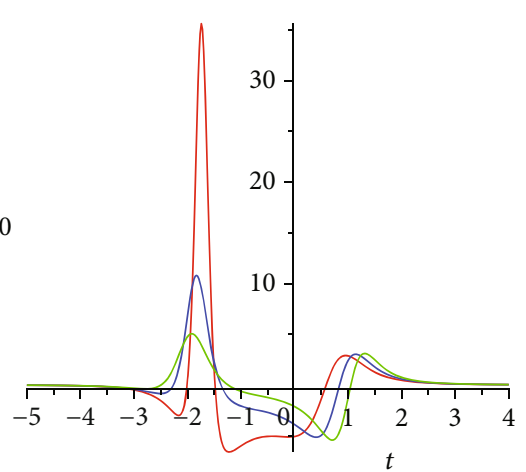

(c)

$$
\begin{aligned}
y & =-1 \\
y & =0 \\
y & =1
\end{aligned}
$$

Figure 9: Plots of interaction with two lumps (52).

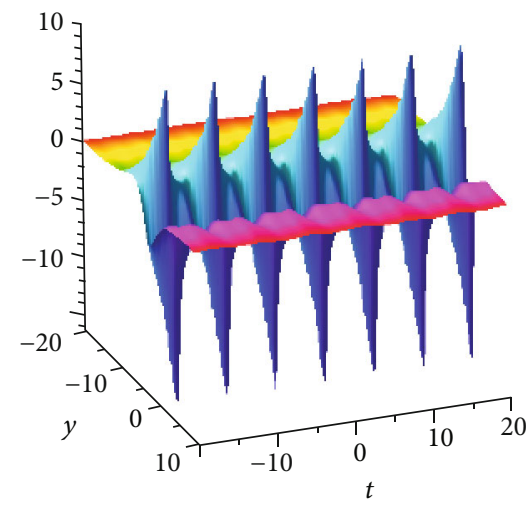

(a)

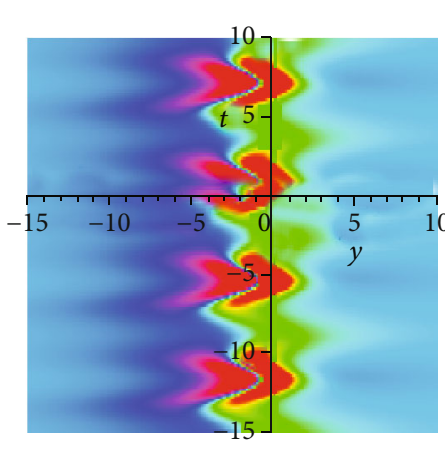

(b)

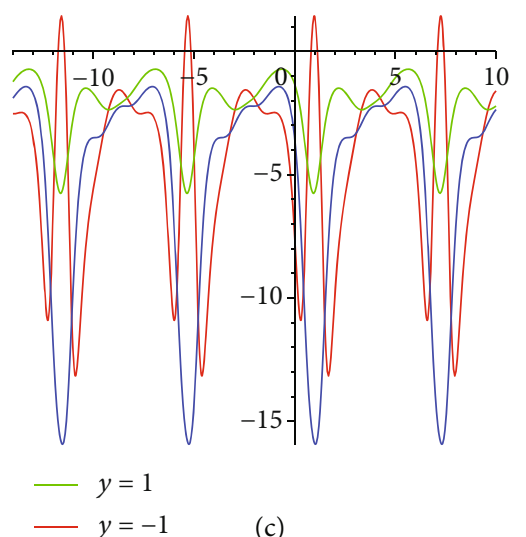

$-y=0$

Figure 10: Plots of interaction with two lumps (52).

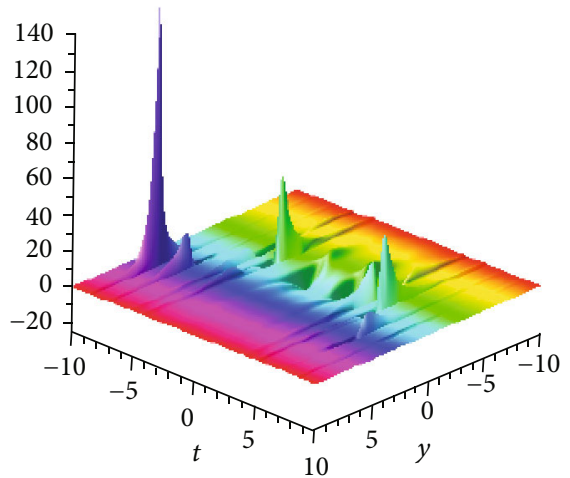

(a)

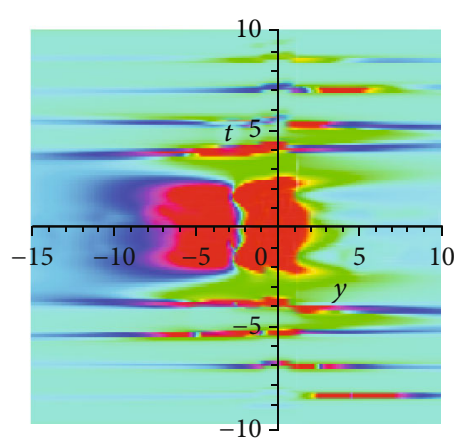

(b)

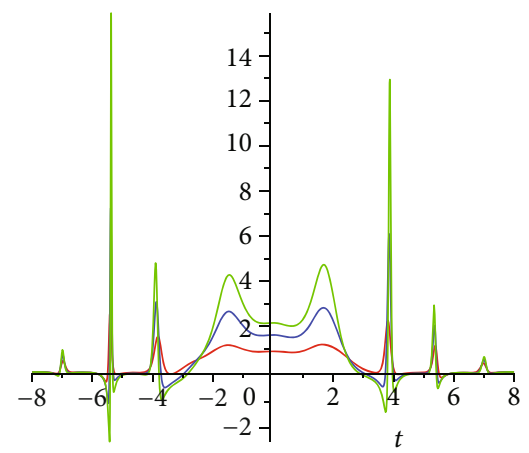

(c)

$y=-8$
$-y=-6$
$y=-5$

FIGURE 11: Plots of interaction with two lumps (52). 
system containing 119 nonlinear equations. To solve the nonlinear system, the determined coefficients will be got as the below cases.

Type I

$$
\begin{aligned}
\varepsilon_{1}(t)= & \frac{\sqrt[4]{\left(A_{1}(t)+A_{2}(t)\right) \alpha_{2}}}{\alpha_{2}}, A_{1}(t) \\
= & 2 \alpha_{3}{ }^{2} \beta_{2}{ }^{2} \int \varepsilon_{2}(t) \phi_{3}(t) d t \\
& -2 \alpha_{3} \int \varepsilon_{3}(t) \phi_{2}(t) d t \alpha_{2}{ }^{3}-2 \alpha_{3} \beta_{2}{ }^{2} \int \varepsilon_{3}(t) \phi_{3}(t) d t \alpha_{2}, \\
A_{2}(t)= & 2 \alpha_{3}{ }^{2} \int \phi_{2}(t) \varepsilon_{2}(t) d t \alpha_{2}{ }^{2}+2 \alpha_{3} \int \varepsilon_{2}(t) \frac{d}{d t} \varepsilon_{3}(t) d t \alpha_{2}{ }^{2} \\
& -\left(\varepsilon_{3}(t)\right)^{2} \alpha_{2}{ }^{3}-\varepsilon_{5}(t) \alpha_{2}{ }^{3}+C_{1} \alpha_{2}{ }^{3}, \\
\varepsilon_{2}(t)= & -\frac{\left(\alpha_{2}{ }^{2}+\alpha_{3}{ }^{2}\right)\left(\phi_{2}(t) \alpha_{2}{ }^{2}+\phi_{3}(t) \beta_{2}{ }^{2}\right)+\left(d / d t \varepsilon_{3}(t)\right) \alpha_{2}{ }^{2} \alpha_{3}}{\alpha_{2}{ }^{3}} d t \\
& +C_{2}, \varepsilon_{4}(t)=0, \\
\alpha_{1}= & \alpha_{4}=\beta_{1}=\beta_{4}=\delta_{1}=\phi_{1}(t)=\phi_{4}(t)=0, \beta_{3}=\frac{\alpha_{3} \beta_{2}}{\alpha_{2}}, \delta_{3}=\frac{\alpha_{3} \delta_{2}}{\alpha_{2}} .
\end{aligned}
$$

The solutions are given as follows:

$$
\begin{aligned}
u_{1}= & 12 \frac{\partial^{2}}{\partial x^{2}} \ln \left(\mathbf{F}_{1}\right), \mathbf{F}_{1}=\left(\varepsilon_{1}(t)\right)^{4}+\left(\alpha_{2} x+\beta_{2} y+\delta_{2} z+\varepsilon_{2}(t)\right)^{2} \\
& +\left(\alpha_{3} x+\frac{\alpha_{3} \beta_{2} y}{\alpha_{2}}+\frac{\alpha_{3} \delta_{2} z}{\alpha_{2}}+\varepsilon_{3}(t)\right)^{2}+e^{\delta_{4} z+\varepsilon_{4}(t)}+\varepsilon_{5}(t) .
\end{aligned}
$$

If $\tau_{1}^{4}+\tau_{2}^{2}+\tau_{3}^{2}+\exp \left(\tau_{4}\right)+\varepsilon_{5}(t) \longrightarrow \infty$, the lump solutions $u \longrightarrow 0$ at any $t$. By selecting the parameters $\delta_{2}=1$, $\delta_{4}=2, \alpha_{2}=1, \alpha_{3}=2, \beta_{2}=3, \phi_{2}(t)=\cos (t), \phi_{3}(t)=\sin (t)$, $\varepsilon_{2}(t)=\cos (2 t), \varepsilon_{3}(t)=\sin (2 t), x=1, y=1$, then plots of equation (59) are plotted in Figure 12. And also, by selecting the parameters $\delta_{2}=1, \delta_{4}=2, \alpha_{2}=1, \alpha_{3}=2, \beta_{2}=3, \phi_{2}(t)=$ $\cos (1+t), \phi_{3}(t)=\sin (1+t), \varepsilon_{2}(t)=\cos (1+3 t), \varepsilon_{3}(t)=\sin$ $(1+3 t), x=1, y=1$, then plots of equation (52) are plotted in Figure 13.

Type II

$$
\begin{aligned}
\varepsilon_{1}(t)= & \frac{\sqrt[4]{\left(A_{1}(t)+A_{2}(t)+A_{3}(t)\right) \alpha_{4}{ }^{2} \alpha_{2}{ }^{3}},}{\alpha_{4} \alpha_{2}}, \\
A_{1}(t)= & 2 \alpha_{3}{ }^{2} \beta_{4}{ }^{2} \int \varepsilon_{2}(t) \phi_{3}(t) d t-2 \alpha_{3} \int \varepsilon_{3}(t) \phi_{2}(t) d t \alpha_{4}{ }^{2} \alpha_{2} \\
& -2 \alpha_{3} \beta_{4}{ }^{2} \int \varepsilon_{3}(t) \phi_{3}(t) d t \alpha_{2}, \\
A_{2}(t)= & 2 \alpha_{3}{ }^{2} \int \phi_{2}(t) \varepsilon_{2}(t) d t \alpha_{4}{ }^{2}+2 \alpha_{3}{ }^{2} \delta_{4}{ }^{2} \int \phi_{4}(t) \varepsilon_{2}(t) d t \\
& -2 \alpha_{3} \delta_{4}{ }^{2} \int \phi_{4}(t) \varepsilon_{3}(t) d t \alpha_{2}, \\
A_{3}(t)= & 2 \alpha_{3} \int \varepsilon_{2}(t) \frac{d}{d t} \varepsilon_{3}(t) d t \alpha_{4}{ }^{2}-\left(\varepsilon_{3}(t)\right)^{2} \alpha_{4}{ }^{2} \alpha_{2}-\varepsilon_{5}(t) \alpha_{4}{ }^{2} \alpha_{2}+C_{1} \alpha_{4}{ }^{2} \alpha_{2}, \\
\varepsilon_{2}(t)= & -\frac{\left(\alpha_{2}{ }^{2}+\alpha_{3}{ }^{2}\right)\left(\alpha_{4}{ }^{2} \phi_{2}(t)+\beta_{4}{ }^{2} \phi_{3}(t)+\delta_{4}{ }^{2} \phi_{4}(t)\right)+\left(d / d t \varepsilon_{3}(t)\right) \alpha_{3} \alpha_{4}{ }^{2}}{\alpha_{4}{ }^{2} \alpha_{2}} d t,
\end{aligned}
$$

$\varepsilon_{4}(t)=\int-\frac{\alpha_{4}{ }^{2} \phi_{2}(t)+\beta_{4}{ }^{2} \phi_{3}(t)+\delta_{4}{ }^{2} \phi_{4}(t)}{\alpha_{4}} d t$

$$
\alpha_{1}=\beta_{1}=\delta_{1}=\phi_{1}(t)=0, \beta_{2}=\frac{\alpha_{2} \beta_{4}}{\alpha_{4}}, \beta_{3}=\frac{\alpha_{3} \beta_{4}}{\alpha_{4}}, \delta_{2}=\frac{\alpha_{2} \delta_{4}}{\alpha_{4}}, \delta_{3}=\frac{\alpha_{3} \delta_{4}}{\alpha_{4}} .
$$

The solutions are given as follows:

$$
\begin{gathered}
u_{2}=12 \frac{\partial^{2}}{\partial x^{2}} \ln \left(\mathbf{F}_{2}\right), \\
\mathbf{F}_{2}=\left(\varepsilon_{1}(t)\right)^{4}+\left(\alpha_{2} x+\frac{\alpha_{2} \beta_{4} y}{\alpha_{4}}+\frac{\alpha_{2} \delta_{4} z}{\alpha_{4}}+\varepsilon_{2}(t)\right)^{2} \\
+\left(\alpha_{3} x+\frac{\alpha_{3} \beta_{4} y}{\alpha_{4}}+\frac{\alpha_{3} \delta_{4} z}{\alpha_{4}}+\varepsilon_{3}(t)\right)^{2} \\
+e^{\alpha_{4} x+\beta_{4} y+\delta_{4} z+\varepsilon_{4}(t)}+\varepsilon_{5}(t) .
\end{gathered}
$$

By selecting the parameters $\delta_{4}=2, \alpha_{2}=1, \alpha_{3}=2, \alpha_{4}=4$, $\beta_{4}=3, \phi_{2}(t)=\cos (t), \phi_{3}(t)=\sin (t), \phi_{4}(t)=\sin (2 t), \varepsilon_{2}(t)$ $=\cos (2 t), \varepsilon_{3}(t)=\sin (2 t), \varepsilon_{4}(t)=\sin (3 t), C_{1}=1, C_{2}=2, x$ $=1, y=1$, then plots of equation (61) are plotted in Figure 14 . And also, by selecting the parameters $\delta_{4}=2, \alpha_{2}=1, \alpha_{3}=$ $2, \alpha_{4}=4, \beta_{4}=3, \phi_{2}(t)=\cos (t), \phi_{3}(t)=\sin (t), \phi_{4}(t)=\sin (2$ $t), \varepsilon_{2}(t)=t, \varepsilon_{3}(t)=t^{2}, \varepsilon_{4}(t)=t^{3}, C_{1}=1, C_{2}=2, x=1, y=1$, then plots of equation (54) are plotted in Figure 15.

4.4. Lump-Periodic Solutions. In this section, we would like to present the general solutions of the including combination of two functions for the $(3+1)$-dimensional variablecoefficient nonlinear wave equation through utilizing the bilinear method as the below frame:

$$
\begin{aligned}
g & =\tau_{1}^{2}+\tau_{2}^{2}+\cos \left(\tau_{3}\right)+\varepsilon_{4}(t), \tau_{j} \\
& =\alpha_{j} x+\beta_{j} y+\delta_{j} z+\varepsilon_{j}(t), j \\
& =1,2,3, \varepsilon_{4}(t)>0 .
\end{aligned}
$$

The values $\alpha_{i}, \beta_{i}, \delta_{i}, \varepsilon_{i}(t)(i=1,2,3)$ are real constants to be computed. By substituting (63) into (22), we obtain a system containing 24 nonlinear equations. To solve the nonlinear system, the determined coefficients will be got as the below cases.

Type I

$$
\begin{aligned}
\varepsilon_{1}(t)= & -\frac{\varepsilon_{2}(t) \alpha_{2}}{\alpha_{1}}-\frac{\alpha_{2}}{\alpha_{1}} \int \frac{\alpha_{1}^{2} \phi_{2}(t)}{\alpha_{2}}+\alpha_{2} \phi_{2}(t) d t+C_{1}, \varepsilon_{3}(t) \\
= & 0, \varepsilon_{4}(t)=-\frac{\left(\alpha_{1}^{2}+\alpha_{2}^{2}\right)\left(\left(\int \phi_{2}(t) d t\right)^{2} \alpha_{2}^{2}+\left(\varepsilon_{2}(t)\right)^{2}\right)}{\alpha_{1}^{2}} \\
& -2 \frac{\varepsilon_{2}(t) \alpha_{2}}{\alpha_{1}{ }^{2}}\left(\int \frac{\phi_{2}(t)\left(\alpha_{1}^{2}+\alpha_{2}^{2}\right)}{\alpha_{2}} d t \alpha_{2}-C_{1} \alpha_{1}\right) \\
& -2 \frac{\int-\phi_{2}(t) C_{1} \alpha_{1} \alpha_{2}{ }^{2} d t}{\alpha_{1}{ }^{2}}+C_{2}, \alpha_{3} \\
= & \phi_{1}(t)=\phi_{3}(t)=0, \beta_{2}=\frac{\alpha_{2} \beta_{1}}{\alpha_{1}}, \delta_{2}=\frac{\alpha_{2} \delta_{1}}{\alpha_{1}} .
\end{aligned}
$$




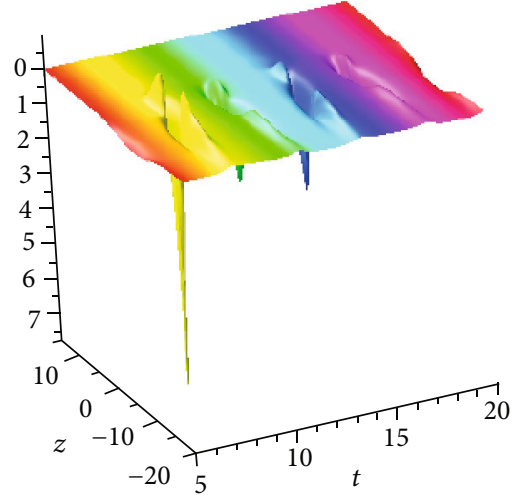

(a)

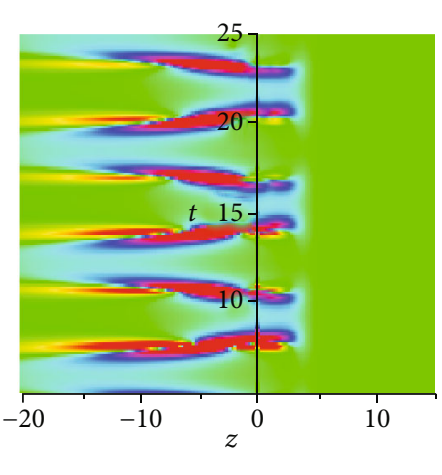

(b)

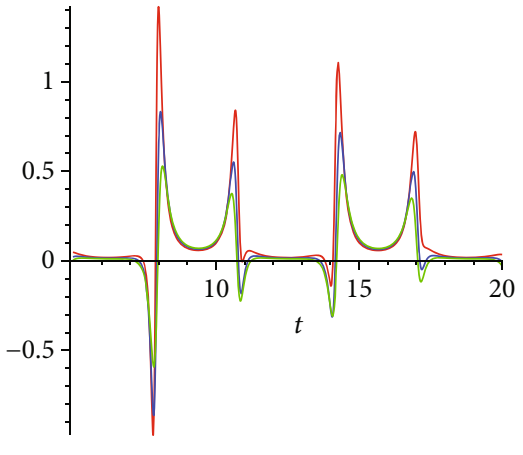

$-z=-1$

$-z=0$

(c)

Figure 12: Plots of interaction with two lumps-soliton (59).

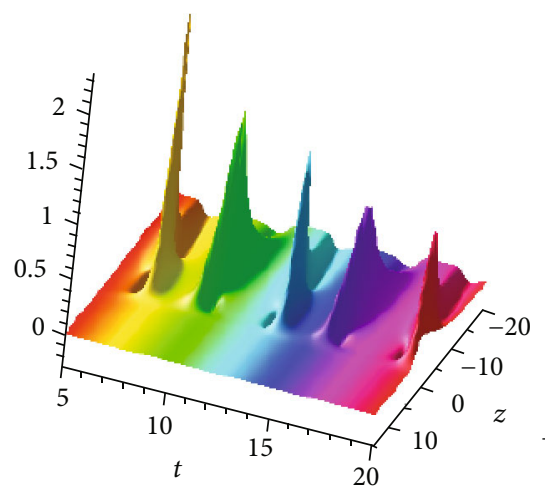

(a)

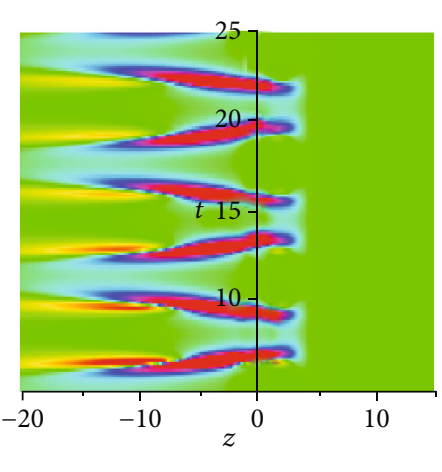

(b)

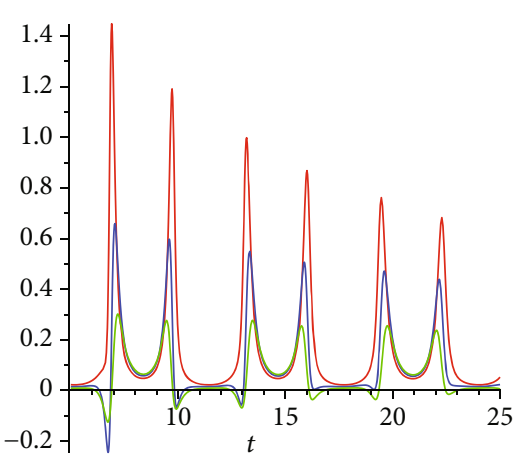

$-z=-2$

$z=0$

(c)

FIGURE 13: Plots of interaction with two lumps-soliton (59).

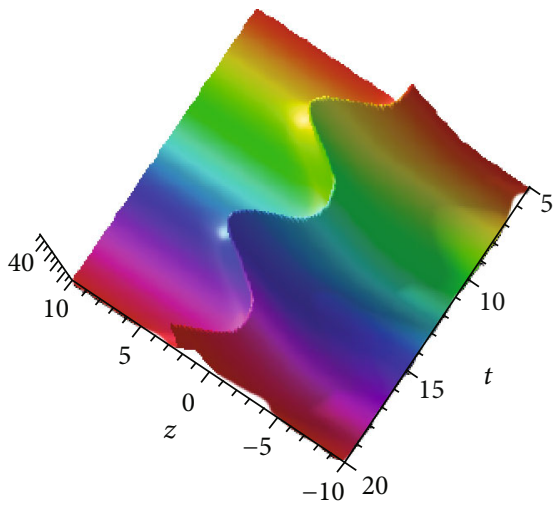

(a)

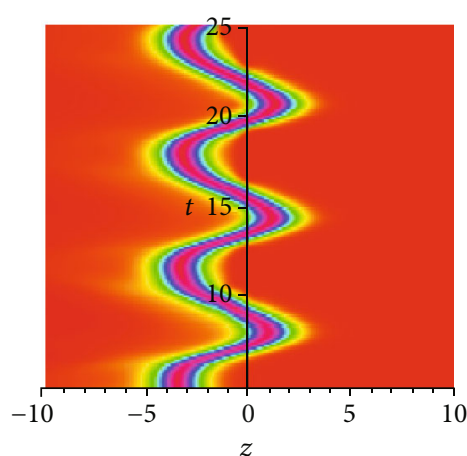

(b)

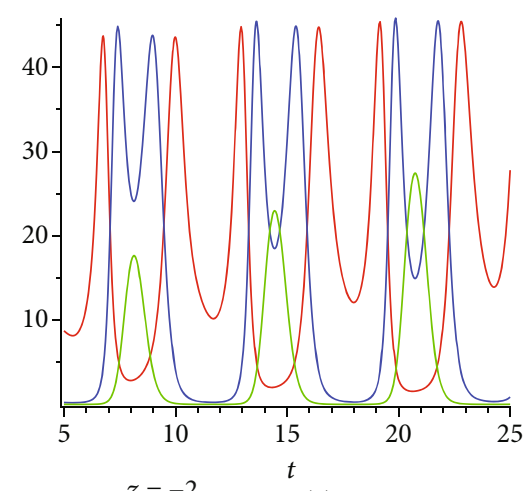

(c)

$\begin{aligned} z & =-2 \\ -z & =0\end{aligned}$

- $z=2$

Figure 14: Plots of interaction with two lumps-soliton (61). 


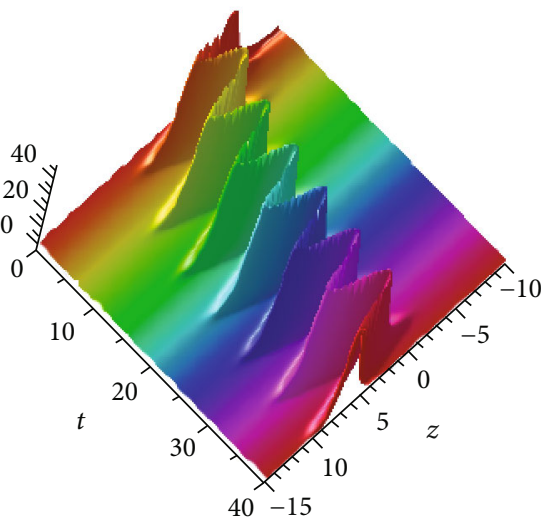

(a)

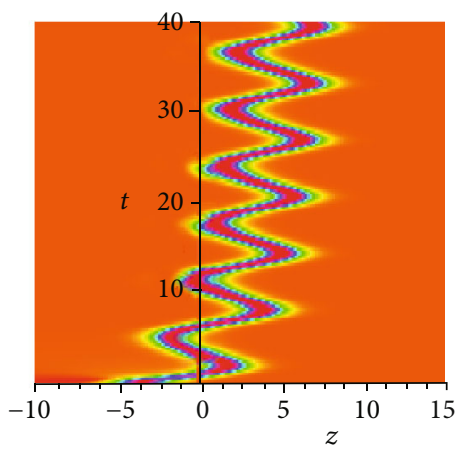

(b)

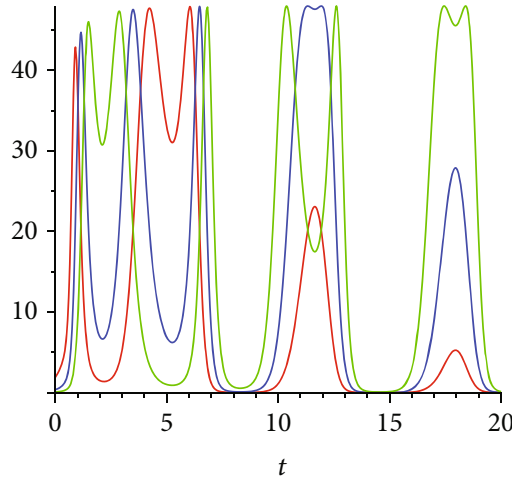

(c)

$-z=-1$

$\begin{aligned} z & =0 \\ z & =1\end{aligned}$

FIGURE 15: Plots of interaction with two lumps-soliton (61).

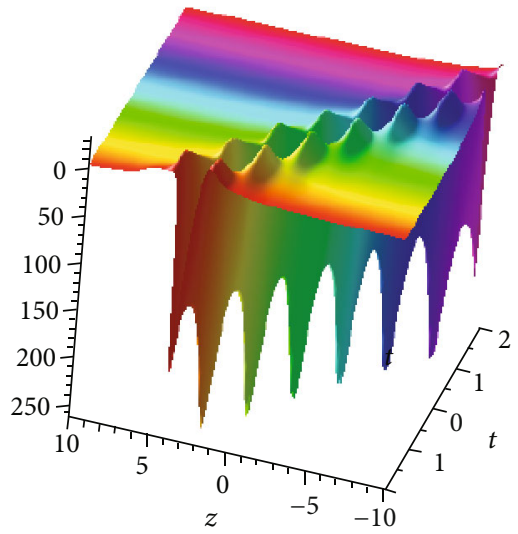

(a)

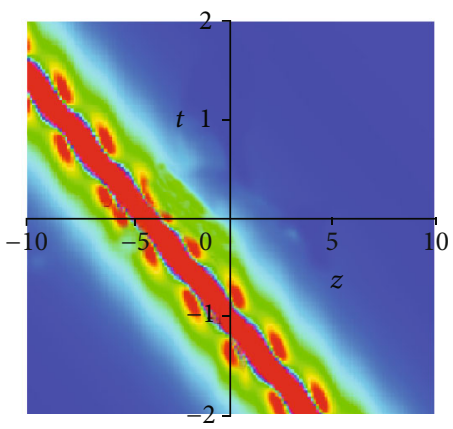

(b)

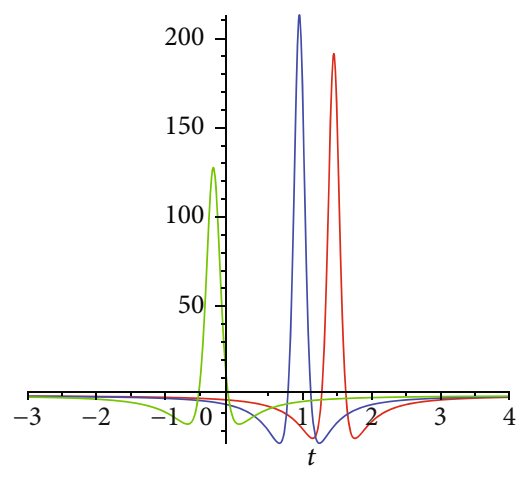

(c)

Figure 16: Plots of lump-periodic (65).

The solutions are given as follows:

$$
\begin{aligned}
u_{1}= & 12 \frac{\partial^{2}}{\partial x^{2}} \ln \left(\mathbf{F}_{1}\right), \mathbf{F}_{1}=\left(\alpha_{1} x+\beta_{1} y+\delta_{1} z+\varepsilon_{1}(t)\right)^{2} \\
& +\left(\alpha_{2} x+\frac{\alpha_{2} \beta_{1} y}{\alpha_{1}}+\frac{\alpha_{2} \delta_{1} z}{\alpha_{1}}+\varepsilon_{2}(t)\right)^{2} \\
& +\cos \left(\beta_{3} y+\delta_{3} z+\varepsilon_{3}(t)\right)+\varepsilon_{4}(t) .
\end{aligned}
$$

From above, we can discover that $g$ is positive if $\varepsilon_{4}(t)$ $>0$, then in all space directions, $u$ will be localized. Therefore, if $\tau_{1}^{2}+\tau_{2}^{2}+\cos \left(\tau_{3}\right) \longrightarrow \infty$, the lump solutions $u \longrightarrow 0$ at any $t$. By selecting the parameters $\delta_{1}=1, \delta_{3}=3, \alpha_{1}=2$, $\alpha_{2}=4, \beta_{1}=2, \beta_{3}=3, \phi_{2}(t)=-2, \varepsilon_{2}(t)=3, C_{1}=1, C_{2}=2, x$ $=1, y=1$, then plots of equation (65) are plotted in Figure 16.
Type II

$$
\begin{aligned}
\varepsilon_{1}(t) & =\int-\frac{\left(\alpha_{1}{ }^{2}+\alpha_{2}{ }^{2}\right)\left(\alpha_{1}{ }^{2} \phi_{2}+\delta_{1}{ }^{2} \phi_{4}\right)+\left(d / d t \varepsilon_{2}(t)\right) \alpha_{1}{ }^{2} \alpha_{2}}{\alpha_{1}{ }^{3}} d t+C_{1}, \varepsilon_{3}(t)=0, \\
\varepsilon_{4}(t) & =\int 2 \frac{\left(\varepsilon_{1}(t) \alpha_{2}-\varepsilon_{2}(t) \alpha_{1}\right)\left(\phi_{2}(t) \alpha_{1}{ }^{2} \alpha_{2}+\phi_{4}(t) \alpha_{2} \delta_{1}{ }^{2}+\left(d / d t \varepsilon_{2}(t)\right) \alpha_{1}{ }^{2}\right)}{\alpha_{1}{ }^{3}} d t+C_{2}, \\
\alpha_{3} & =\delta_{3}=\phi_{1}(t)=\phi_{3}(t)=0, \beta_{2}=\frac{\alpha_{2} \beta_{1}}{\alpha_{1}}, \delta_{2}=\frac{\alpha_{2} \delta_{1}}{\alpha_{1}} .
\end{aligned}
$$

The solutions are given as follows:

$$
\begin{aligned}
u_{2}= & 12 \frac{\partial^{2}}{\partial x^{2}} \ln \left(\mathbf{F}_{2}\right), \mathbf{F}_{2}=\left(\alpha_{1} x+\beta_{1} y+\delta_{1} z+\varepsilon_{1}(t)\right)^{2} \\
& +\left(\alpha_{2} x+\frac{\alpha_{2} \beta_{1} y}{\alpha_{1}}+\frac{\alpha_{2} \delta_{1} z}{\alpha_{1}}+\varepsilon_{2}(t)\right)^{2} \\
& +\cos \left(\beta_{3} y+\varepsilon_{3}(t)\right)+\varepsilon_{4}(t) .
\end{aligned}
$$


Type III

$$
\begin{aligned}
\varepsilon_{1}(t) & =\int-2 \frac{\delta_{1} \phi_{4}(t)\left(\beta_{1} \delta_{2}-\beta_{2} \delta_{1}\right)}{\alpha_{2} \beta_{1}} d t, \varepsilon_{2}(t) \\
& =\int-\frac{\alpha_{2}{ }^{2} \beta_{1}{ }^{2} \phi_{2}(t)+\beta_{1}{ }^{2} \delta_{2}{ }^{2} \phi_{4}(t)-\beta_{2}{ }^{2} \delta_{1}{ }^{2} \phi_{4}(t)}{\alpha_{2} \beta_{1}{ }^{2}} d t, \\
\varepsilon_{3}(t) & =\int-2 \frac{\delta_{3} \phi_{4}(t)\left(\beta_{1} \delta_{2}-\beta_{2} \delta_{1}\right)}{\alpha_{2} \beta_{1}} d t, \varepsilon_{4}(t) \\
& =0, \beta_{3}=\frac{\beta_{1} \delta_{3}}{\delta_{1}}, \phi_{3}(t)=-\frac{\delta_{1}{ }^{2} \phi_{4}(t)}{\beta_{1}{ }^{2}}, \\
\alpha_{1} & =\alpha_{3}=\phi_{1}(t)=0, \beta_{2}=\frac{\alpha_{2} \beta_{1}}{\alpha_{1}}, \delta_{2}=\frac{\alpha_{2} \delta_{1}}{\alpha_{1}} .
\end{aligned}
$$

The solutions are given as follows:

$$
\begin{aligned}
u_{3}= & 12 \frac{\partial^{2}}{\partial x^{2}} \ln \left(\mathbf{F}_{3}\right), \mathbf{F}_{3}=\left(\beta_{1} y+\delta_{1} z+\varepsilon_{1}(t)\right)^{2} \\
& +\left(\alpha_{2} x+\beta_{2} y+\delta_{2} z+\varepsilon_{2}(t)\right)^{2} \\
& +\cos \left(\frac{\beta_{1} \delta_{3} y}{\delta_{1}}+\delta_{3} z+\varepsilon_{3}(t)\right)+\varepsilon_{4}(t) .
\end{aligned}
$$

By selecting the parameters $\delta_{1}=1, \delta_{2}=2, \delta_{3}=3, \alpha_{2}=4$, $\beta_{1}=2, \beta_{2}=3, \phi_{2}(t)=\sin (t), \phi_{4}(t)=\cos (t), x=1, y=1$, then plots of equation (69) are plotted in Figure 17.

Type IV

$$
\begin{aligned}
\varepsilon_{2}(t) & =-\tan \left(\alpha_{2} \int \frac{\phi_{2}(t)}{\varepsilon_{1}(t)} d t+C_{1}\right) \varepsilon_{1}, \varepsilon_{4}(t) \\
& =\int 2 \frac{\left(\left(\varepsilon_{1}(t)\right)^{2}+\left(\varepsilon_{2}(t)\right)^{2}\right)\left(\alpha_{2} \phi_{2}(t) \varepsilon_{2}(t)-\left(d / d t \varepsilon_{1}(t)\right) \varepsilon_{1}(t)\right)}{\left(\varepsilon_{1}(t)\right)^{2}} d t+C_{2}, \\
\varepsilon_{3}(t) & =0, \alpha_{1}=-\frac{\varepsilon_{2}(t) \alpha_{2}}{\varepsilon_{1}(t)}, \alpha_{3}=\beta_{1}=\beta_{2}=\delta_{1}=\delta_{2} \\
& =\phi_{1}(t)=0, \phi_{3}(t)=-\frac{\delta_{3}{ }^{2} \phi_{4}(t)}{\beta_{3}{ }^{2}} .
\end{aligned}
$$

The solutions are given as follows:

$$
\begin{aligned}
u_{4}= & 12 \frac{\partial^{2}}{\partial x^{2}} \ln \left(\mathbf{F}_{4}\right), \mathbf{F}_{4}=\left(-\frac{\varepsilon_{2}(t) \alpha_{2} x}{\varepsilon_{1}(t)}+\varepsilon_{1}(t)\right)^{2} \\
& +\left(\alpha_{2} x+\varepsilon_{2}(t)\right)^{2}+\cos \left(\beta_{3} y+\delta_{3} z+\varepsilon_{3}(t)\right)+\varepsilon_{4}(t) .
\end{aligned}
$$

Type $V$

$$
\begin{aligned}
\varepsilon_{2}(t) & =-\tan \left(\frac{1}{\alpha_{2} \delta_{3}{ }^{2}} \int \frac{\alpha_{2}{ }^{2} \delta_{3}{ }^{2} \phi_{2}(t)-\beta_{3}{ }^{2} \delta_{2}{ }^{2} \phi_{3}(t)}{\varepsilon_{1}(t)} d t\right) \varepsilon_{1}(t), \varepsilon_{3}(t)=\int 2 \frac{\beta_{3}{ }^{2} \delta_{2} \phi_{3}(t)}{\delta_{3} \alpha_{2}} d t, \\
\varepsilon_{4}(t) & =\int 2 \frac{\left(\left(\varepsilon_{1}(t)\right)^{2}+\left(\varepsilon_{2}(t)\right)^{2}\right)\left(\phi_{2}(t) \varepsilon_{2}(t) \alpha_{2}{ }^{2} \delta_{3}{ }^{2}-\phi_{3}(t) \varepsilon_{2}(t) \beta_{3}{ }^{2} \delta_{2}{ }^{2}-\left(d / d t \varepsilon_{1}(t)\right) \alpha_{2} \delta_{3}{ }^{2} \varepsilon_{1}(t)\right)}{\alpha_{2} \delta_{3}{ }^{2}\left(\varepsilon_{1}(t)\right)^{2}} d t, \\
\alpha_{3} & =\beta_{1}=\beta_{2}=\phi_{1}(t)=0, \alpha_{1}=-\frac{\alpha_{2} \varepsilon_{2}(t)}{\varepsilon_{1}(t)}, \delta_{1}=-\frac{\delta_{2} \varepsilon_{2}(t)}{\varepsilon_{1}(t)}, \phi_{4}(t)=-\frac{\beta_{3}{ }^{2} \phi_{3}(t)}{\delta_{3}{ }^{2}} .
\end{aligned}
$$

The solutions are given as follows:

$u_{5}=12 \frac{\partial^{2}}{\partial x^{2}} \ln \left(\mathbf{F}_{5}\right), \mathbf{F}_{5}=\left(-\frac{\alpha_{2} \varepsilon_{2}(t) x}{\varepsilon_{1}(t)}-\frac{\delta_{2} \varepsilon_{2}(t) z}{\varepsilon_{1}(t)}+\varepsilon_{1}(t)\right)^{2}+\left(\alpha_{2} x+\delta_{2} z+\varepsilon_{2}(t)\right)^{2}+\cos \left(\beta_{3} y+\delta_{3} z+\varepsilon_{3}(t)\right)+\varepsilon_{4}(t)$.

Type VI

$$
\begin{aligned}
\varepsilon_{3}(t) & =0, \varepsilon_{4}(t)=-\left(\varepsilon_{1}(t)\right)^{2}-\left(\varepsilon_{2}(t)\right)^{2}, \\
\alpha_{3} & =\beta_{1}=\beta_{2}=\phi_{1}(t)=\phi_{3}(t)=0, \alpha_{1}=-\frac{\alpha_{2} \varepsilon_{2}(t)}{\varepsilon_{1}(t)}, \delta_{1}=-\frac{\delta_{2} \varepsilon_{2}(t)}{\varepsilon_{1}(t)}, \phi_{4}(t)=-\frac{\beta_{3}{ }^{2} \phi_{3}(t)}{\delta_{3}{ }^{2}}, \\
\phi_{4}(t) & =\frac{\left(-\alpha_{2} \phi_{2}(t)\left(\left(\varepsilon_{1}(t)\right)^{2}+\left(\varepsilon_{2}(t)\right)^{2}\right)+\left(d / d t \varepsilon_{1}(t)\right) \varepsilon_{1}(t) \varepsilon_{2}(t)-\left(d / d t \varepsilon_{2}(t)\right)\left(\varepsilon_{1}(t)\right)^{2}\right) \alpha_{2}}{\left(\left(\varepsilon_{1}(t)\right)^{2}+\left(\varepsilon_{2}(t)\right)^{2}\right) \delta_{2}{ }^{2}} .
\end{aligned}
$$




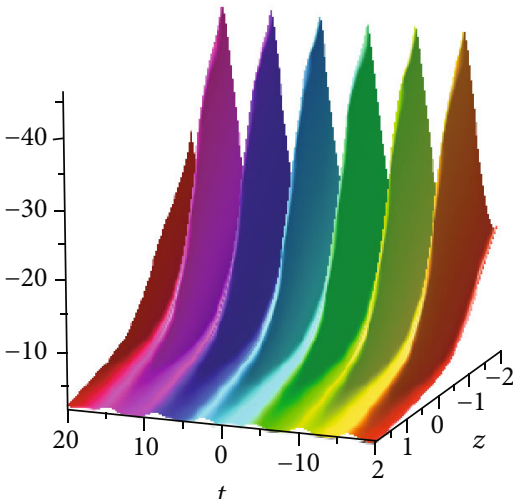

(a)

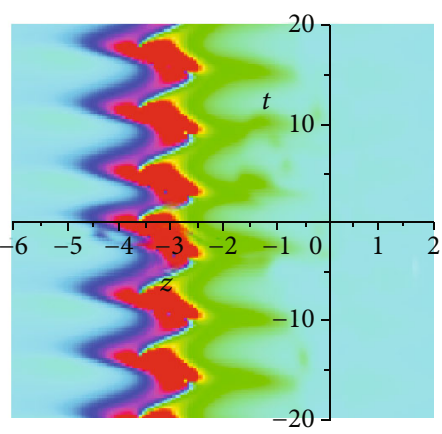

(b)

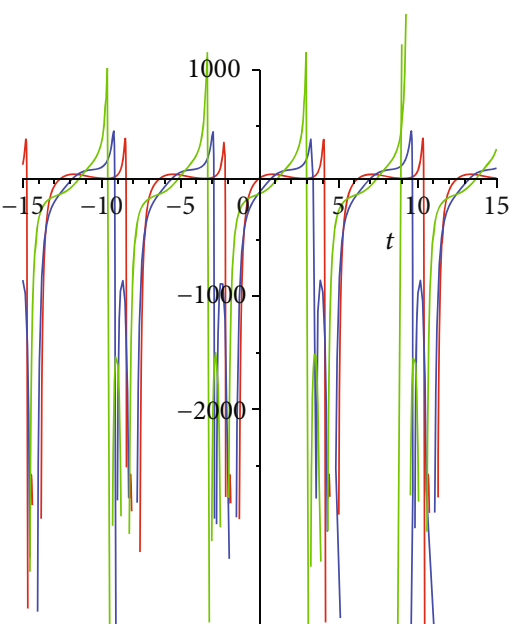

$-z=-3.5$

$z=-3.2$

$z=-3$

(c)

Figure 17: Plots of lump-periodic (69).

The solutions are given as follows:

$$
\begin{aligned}
u_{6}= & 12 \frac{\partial^{2}}{\partial x^{2}} \ln \left(\mathbf{F}_{6}\right), \mathbf{F}_{6}=\left(-\frac{\alpha_{2} \varepsilon_{2}(t) x}{\varepsilon_{1}(t)}-\frac{\delta_{2} \varepsilon_{2}(t) z}{\varepsilon_{1}(t)}+\varepsilon_{1}(t)\right)^{2} \\
& +\left(\alpha_{2} x+\delta_{2} z+\varepsilon_{2}(t)\right)^{2}+\cos \left(\beta_{3} y\right)-\left(\varepsilon_{1}(t)\right)^{2}-\left(\varepsilon_{2}(t)\right)^{2} .
\end{aligned}
$$

Type VII

$\varepsilon_{1}(t)=\int-\frac{\phi_{2}(t)\left(\alpha_{1}^{2}+\alpha_{2}^{2}\right)+\left(d / d t \varepsilon_{2}(t)\right) \alpha_{2}}{\alpha_{1}} d t+C_{1}, \varepsilon_{3}(t)=0$,

$\varepsilon_{4}(t)=\int-2 \frac{\alpha_{2} \phi_{2}(t)\left(\alpha_{1} \varepsilon_{2}(t)-\alpha_{2} \varepsilon_{1}(t)\right)+\left(d / d t \varepsilon_{2}(t)\right)\left(\alpha_{1} \varepsilon_{2}(t)-\alpha_{2} \varepsilon_{1}(t)\right)}{\alpha_{1}} d t+C_{2}$,

$\alpha_{3}=\beta_{1}=\beta_{2}=\beta_{3}=\phi_{1}(t)=\phi_{4}(t)=0, \delta_{2}=\frac{\alpha_{2} \delta_{1}}{\alpha_{1}}$.

The solutions are given as follows:

$$
\begin{aligned}
u_{7}= & 12 \frac{\partial^{2}}{\partial x^{2}} \ln \left(\mathbf{F}_{7}\right), \mathbf{F}_{7}=\left(\alpha_{1} x+\delta_{1} z+\varepsilon_{1}(t)\right)^{2} \\
& +\left(\alpha_{2} x+\frac{\alpha_{2} \delta_{1} z}{\alpha_{1}}+\varepsilon_{2}(t)\right)^{2}+\cos \left(\delta_{3} z+\varepsilon_{3}(t)\right)+\varepsilon_{4}(t)
\end{aligned}
$$

By selecting the parameters $\delta_{1}=2, \alpha_{1}=1, \alpha_{2}=2, \beta_{3}=1$, $\phi_{2}(t)=t, \phi_{4}(t)=t^{2}, \varepsilon_{1}(t)=t, \varepsilon_{2}(t)=1+t, \varepsilon_{3}(t)=t^{2}, x=1, y$ $=1$, then plots of equation (77) are plotted in Figure 18. And also, by selecting the parameters $\delta_{1}=2, \alpha_{1}=1, \alpha_{2}=2, \beta_{3}$ $=1, \phi_{2}(t)=\sin (t), \phi_{4}(t)=\cos (t), \varepsilon_{1}(t)=t, \varepsilon_{2}(t)=\sin (t)$ , $\varepsilon_{3}(t)=\cos (t), x=1, y=1$, then plots of equation (77) are plotted in Figure 19. Moreover, by selecting the parameters $\delta_{1}=2, \alpha_{1}=1, \alpha_{2}=2, \beta_{3}=1, \phi_{2}(t)=1, \phi_{4}(t)=2, \varepsilon_{1}(t)=$ $t, \varepsilon_{2}(t)=t, \varepsilon_{3}(t)=t^{2}, x=1, y=1$, then plots of equation (77) are plotted in Figure 20. And finally, by selecting the parameters $\delta_{1}=2, \alpha_{1}=1, \alpha_{2}=2, \beta_{3}=1, \phi_{2}(t)=\exp (t), \phi_{4}(t)$ $=\exp (2 t), \varepsilon_{1}(t)=\sinh (t), \varepsilon_{2}(t)=\cosh (t), \varepsilon_{3}(t)=t^{2}, x=1$, $y=1$, then plots of equation (77) are plotted in Figure 21 .

4.5. Lump-Three Kink Solutions. Here, we present lumpperiodic solutions containing combination of two functions for the $(3+1)$-dimensional variable-coefficient nonlinear wave equation through utilizing the bilinear method as the below frame:

$$
\begin{aligned}
g & =\tau_{1}^{2}+\tau_{2}^{2}+\exp \left(\tau_{3}\right)+\exp \left(\tau_{4}\right)+\exp \left(\tau_{5}\right)+\varepsilon_{6}(t), \tau_{j} \\
& =\alpha_{j} x+\beta_{j} y+\delta_{j} z+\varepsilon_{j}(t), j=1,2,3,4,5, \varepsilon_{6}(t)>0 .
\end{aligned}
$$

The values $\alpha_{i}, \beta_{i}, \delta_{i}, \varepsilon_{i}(t)(i=1, \cdots, 5)$ are real constants to be computed. By appending (78) into (22), we receive a system containing 43 nonlinear equations. To solve the nonlinear system, the determined coefficients will be got as the below cases.

Type I

$$
\begin{aligned}
\varepsilon_{1}(t) & =\int-2 \frac{\beta_{5} \delta_{1} \phi_{3}(t)\left(\beta_{2} \delta_{5}-\beta_{5} \delta_{2}\right)}{\delta_{5}{ }^{2} \alpha_{2}} d t, \varepsilon_{2}(t) \\
& =\int-\frac{\alpha_{2}{ }^{2} \delta_{5}{ }^{2} \phi_{2}(t)+\beta_{2}{ }^{2} \delta_{5}{ }^{2} \phi_{3}(t)-\beta_{5}{ }^{2} \delta_{2}{ }^{2} \phi_{3}(t)}{\delta_{5}{ }^{2} \alpha_{2}} d t \\
\varepsilon_{3}(t) & =\int-2 \frac{\beta_{5} \delta_{3} \phi_{3}(t)\left(\beta_{2} \delta_{5}-\beta_{5} \delta_{2}\right)}{\delta_{5}^{2} \alpha_{2}} d t, \varepsilon_{4}(t) \\
& =\int-2 \frac{\beta_{5} \delta_{4} \phi_{3}(t)\left(\beta_{2} \delta_{5}-\beta_{5} \delta_{2}\right)}{\delta_{5}{ }^{2} \alpha_{2}} d t
\end{aligned}
$$




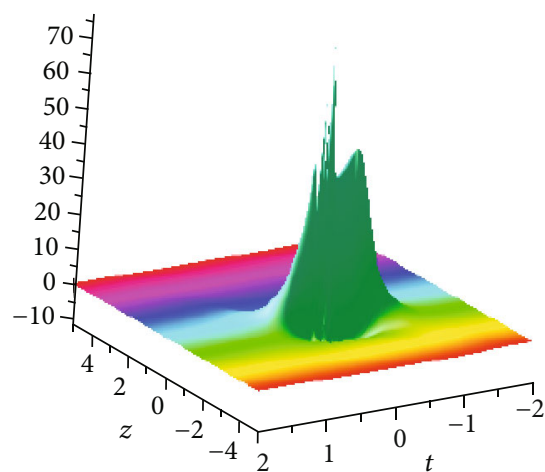

(a)

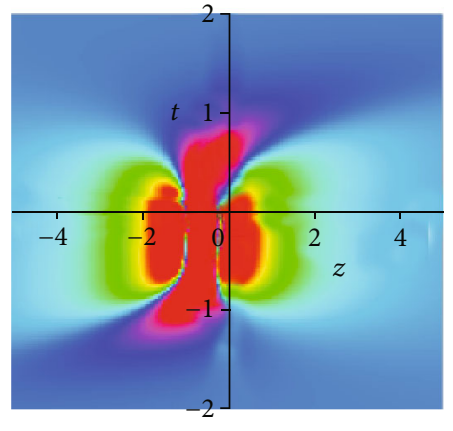

(b)

FIGURE 18: Plots of lump-periodic (77).

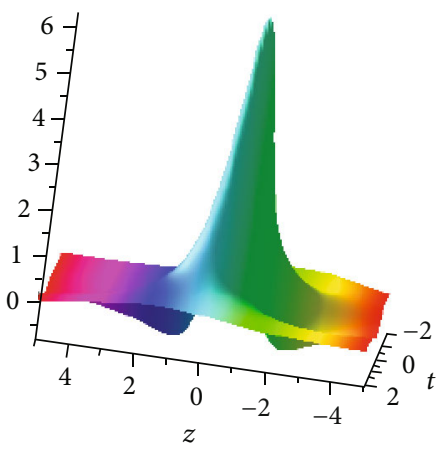

(a)

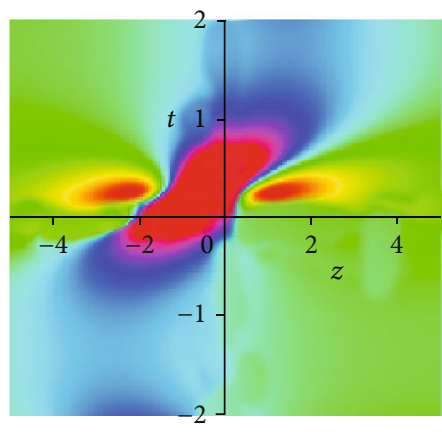

(b)

FIgURE 19: Plots of lump-periodic (77).

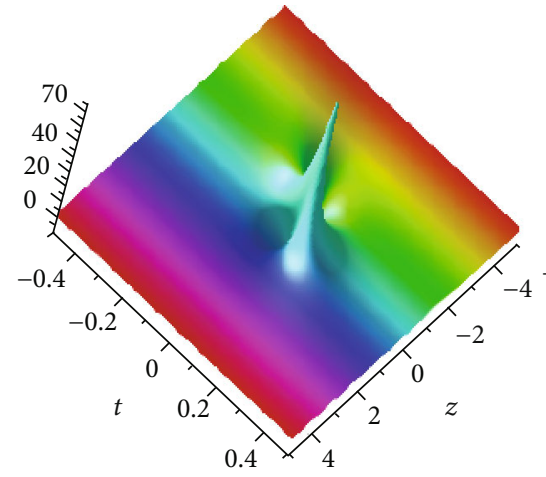

(a)

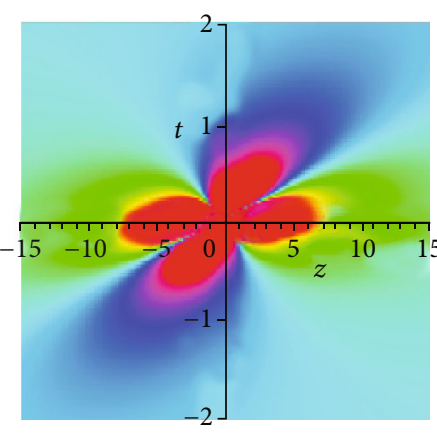

(b)

Figure 20: Plots of lump-periodic (77).

$$
\begin{aligned}
\varepsilon_{5}(t) & =\int-2 \frac{\beta_{5} \phi_{3}(t)\left(\beta_{2} \delta_{5}-\beta_{5} \delta_{2}\right)}{\alpha_{2} \delta_{5}} d t, \varepsilon_{6}(t) \\
& =0, \alpha_{1}=\alpha_{3}=\alpha_{4}=\alpha_{5}=\phi_{1}(t)=0, \beta_{1}=\frac{\delta_{1} \beta_{5}}{\delta_{5}}, \\
\beta_{3} & =\frac{\beta_{5} \delta_{3}}{\delta_{5}}, \beta_{4}=\frac{\beta_{5} \delta_{4}}{\delta_{5}}, \phi_{4}(t)=-\frac{\beta_{5}^{2} \phi_{3}(t)}{\delta_{5}^{2}}
\end{aligned}
$$

The solutions are given as follows:

$$
\begin{aligned}
u_{1}= & 12 \frac{\partial^{2}}{\partial x^{2}} \ln \left(\mathbf{F}_{1}\right), \mathbf{F}_{1}=\left(\frac{\delta_{1} \beta_{5} y}{\delta_{5}}+\delta_{1} z+\varepsilon_{1}(t)\right)^{2} \\
& +\left(\alpha_{2} x+\beta_{2} y+\delta_{2} z+\varepsilon_{2}(t)\right)^{2}+e^{\beta_{5} \delta_{3} y / \delta_{5}+\delta_{3} z+\varepsilon_{3}(t)} \\
& +e^{\beta_{5} \delta_{4} y / \delta_{5}+\delta_{4} z+\varepsilon_{4}(t)}+e^{\beta_{5} y+\delta_{5} z+\varepsilon_{5}(t)}+\varepsilon_{6}(t) .
\end{aligned}
$$




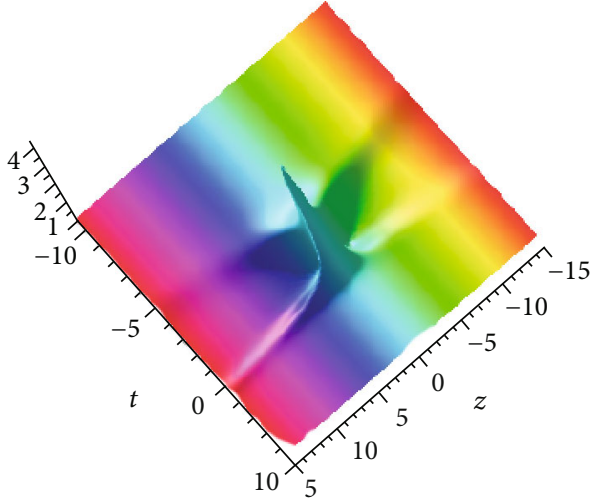

(a)

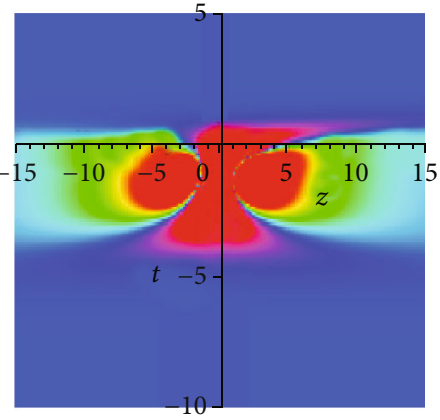

(b)

FIgURe 21: Plots of lump-periodic (77).

Type II

$$
\begin{aligned}
& \varepsilon_{1}(t)=\int-\frac{\left(\alpha_{1}{ }^{2}+\alpha_{2}{ }^{2}\right)\left(\alpha_{4}{ }^{2} \phi_{2}(t)+\beta_{4}{ }^{2} \phi_{3}(t)+\delta_{4}{ }^{2} \phi_{4}(t)\right)+\left(d / d t \varepsilon_{2}(t)\right) \alpha_{2} \alpha_{4}{ }^{2}}{\alpha_{1} \alpha_{4}{ }^{2}} d t \\
& \varepsilon_{3}(t)=\int-\frac{\alpha_{3}\left(\alpha_{4}{ }^{2} \phi_{2}(t)+\beta_{4}{ }^{2} \phi_{3}(t)+\delta_{4}{ }^{2} \phi_{4}(t)\right)}{\alpha_{4}{ }^{2}} d t, \varepsilon_{4}(t)=\int-\frac{\alpha_{4}{ }^{2} \phi_{2}(t)+\beta_{4}{ }^{2} \phi_{3}(t)+\delta_{4}{ }^{2} \phi_{4}(t)}{\alpha_{4}} d t \\
& \varepsilon_{5}(t)=\int-\frac{\alpha_{5}\left(\alpha_{4}{ }^{2} \phi_{2}(t)+\beta_{4}{ }^{2} \phi_{3}(t)+\delta_{4}{ }^{2} \phi_{4}(t)\right)}{\alpha_{4}{ }^{2}} d t, \phi_{1}(t)=0, \beta_{i}=\frac{\alpha_{i} \beta_{4}}{\alpha_{4}}, \delta_{i}=\frac{\alpha_{i} \delta_{4}}{\alpha_{4}}, i=1,2,3,5, \\
& \varepsilon_{6}(t)=\int-2 \frac{\alpha_{2}\left(\alpha_{4}{ }^{2} \phi_{2}(t)+\beta_{4}{ }^{2} \phi_{3}(t)+\delta_{4}{ }^{2} \phi_{4}(t)\right)\left(\alpha_{1} \varepsilon_{2}(t)-\alpha_{2} \varepsilon_{1}(t)\right)+\left(d / d t \varepsilon_{2}(t)\right) \alpha_{4}{ }^{2}\left(\alpha_{1} \varepsilon_{2}(t)-\alpha_{2} \varepsilon_{1}(t)\right)}{\alpha_{1} \alpha_{4}{ }^{2}} d t
\end{aligned}
$$

The solutions are given as follows:

$$
\begin{aligned}
u_{2}= & 12 \frac{\partial^{2}}{\partial x^{2}} \ln \left(\mathbf{F}_{2}\right), \mathbf{F}_{2}=\left(\alpha_{1} x+\frac{\alpha_{1} \beta_{4} y}{\alpha_{4}}+\frac{\alpha_{1} \delta_{4} z}{\alpha_{4}}+\varepsilon_{1}(t)\right)^{2} \\
& +\left(\alpha_{2} x+\frac{\alpha_{2} \beta_{4} y}{\alpha_{4}}+\frac{\alpha_{2} \delta_{4} z}{\alpha_{4}}+\varepsilon_{2}(t)\right)^{2} \\
& +e^{\alpha_{3} x+\alpha_{3} \beta_{4} y / \alpha_{4}+\alpha_{3} \delta_{4} z / \alpha_{4}+\varepsilon_{3}(t)}+e^{\alpha_{4} x+\beta_{4} y+\delta_{4} z+\varepsilon_{4}(t)} \\
& +e^{\alpha_{5} x+\alpha_{5} \beta_{4} y / \alpha_{4}+\alpha_{5} \delta_{4} z / \alpha_{4}+\varepsilon_{5}(t)}+\varepsilon_{6}(t) .
\end{aligned}
$$

$$
\begin{aligned}
\varepsilon_{5}(t) & =\int-\alpha_{5} \phi_{2}(t) d t, \varepsilon_{6}(t) \\
& =\int-2 \frac{\left(\alpha_{1} \varepsilon_{2}(t)-\alpha_{2} \varepsilon_{1}(t)\right)\left(\alpha_{2} \phi_{2}(t)+d / d t \varepsilon_{2}(t)\right)}{\alpha_{1}} d t, \\
\beta_{2} & =\frac{\alpha_{2} \beta_{1}}{\alpha_{1}}, \beta_{3}=\frac{\beta_{1} \delta_{3}}{\delta_{1}}, \delta_{2}=\frac{\alpha_{2} \delta_{1}}{\alpha_{1}}, \delta_{4}=\frac{\beta_{4} \delta_{1}}{\beta_{1}}, \delta_{5} \\
& =\frac{\delta_{1} \beta_{5}}{\beta_{1}}, \phi_{1}(t)=0, \phi_{4}(t)=-\frac{\beta_{1}{ }^{2} \phi_{3}(t)}{\delta_{1}{ }^{2}} .
\end{aligned}
$$

The solutions are given as follows:

Type III

$$
\begin{aligned}
\varepsilon_{1}(t) & =\int-\frac{\phi_{2}(t)\left(\alpha_{1}^{2}+\alpha_{2}^{2}\right)}{\alpha_{1}} d t-\frac{\varepsilon_{2}(t) \alpha_{2}}{\alpha_{1}}, \varepsilon_{3}(t) \\
& =\int-\alpha_{3} \phi_{2}(t) d t, \varepsilon_{4}(t)=\int-\alpha_{4} \phi_{2}(t) d t
\end{aligned}
$$

$$
\begin{aligned}
u_{3}= & 12 \frac{\partial^{2}}{\partial x^{2}} \ln \left(\mathbf{F}_{3}\right), \mathbf{F}_{3}=\left(\alpha_{1} x+\beta_{1} y+\delta_{1} z+\varepsilon_{1}(t)\right)^{2} \\
& +\left(\alpha_{2} x+\frac{\alpha_{2} \beta_{1} y}{\alpha_{1}}+\frac{\alpha_{2} \delta_{1} z}{\alpha_{1}}+\varepsilon_{2}(t)\right)^{2} \\
& +e^{\alpha_{3} x+\beta_{1} \delta_{3} y / \delta_{1}+\delta_{3} z+\varepsilon_{3}(t)}+e^{\alpha_{4} x+\beta_{4} y+\beta_{4} \delta_{1} z / \beta_{1}+\varepsilon_{4}(t)} \\
& +e^{\alpha_{5} x+\beta_{5} y+\delta_{1} \beta_{5} z / \beta_{1}+\varepsilon_{5}(t)}+\varepsilon_{6}(t) .
\end{aligned}
$$




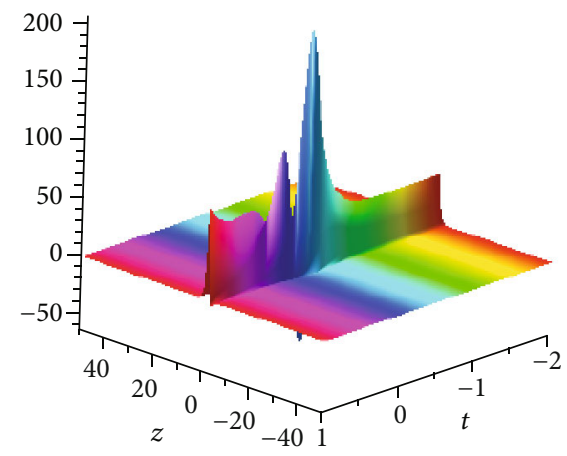

(a)

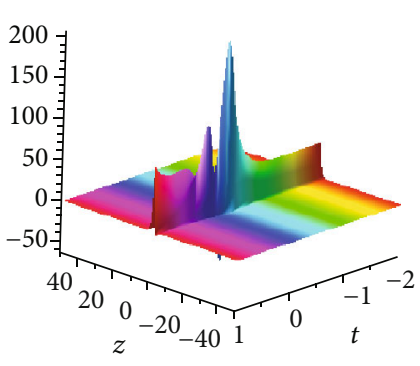

(b)

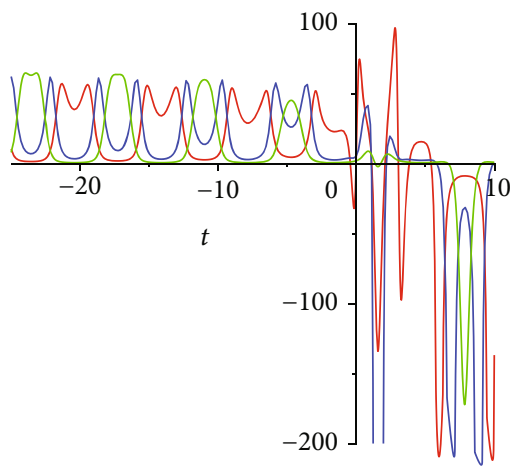

(c)

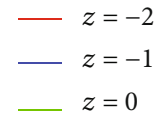

FIGURE 22: Plots of lump-three kink solutions (84).

From above, we can discover that $g$ is positive if $\varepsilon_{6}$ $(t)>0$, then in all space directions $u$ will be localized. Therefore, if $\tau_{1}^{2}+\tau_{2}^{2}+\exp \left(\tau_{3}\right)+\exp \left(\tau_{4}\right)+\exp \left(\tau_{5}\right)+\varepsilon_{6}(t)$ $\longrightarrow \infty$, the lump-three kink solutions

$$
u \Rightarrow \begin{cases}-12 \alpha_{3}^{2}, & \alpha_{3}>\alpha_{4}>\alpha_{5}, \\ 0, & \alpha_{4}>\alpha_{3}>\alpha_{3}, \\ 0, & \alpha_{5}>\alpha_{3}>\alpha_{4}, \\ 0, & \alpha_{4}>\alpha_{5}>\alpha_{3},\end{cases}
$$

at any $t$. By selecting the parameters $\alpha_{1}=1, \alpha_{2}=2, \alpha_{3}=$ $3, \alpha_{4}=4, \alpha_{5}=5, \beta_{1}=1, \beta_{4}=3, \beta_{5}=4, \delta_{1}=1, \delta_{3}=1.5, \phi_{2}(t)$ $=\cos (t), \varepsilon_{1}(t)=\sin (2 t), \varepsilon_{2}(t)=\cos (2 t), x=1, y=1$, then plots of equation (84) are plotted in Figure 22. And also, via selecting the parameters $\alpha_{1}=1, \alpha_{2}=2, \alpha_{3}=3, \alpha_{4}$ $=4, \alpha_{5}=5, \beta_{1}=1, \beta_{4}=3, \beta_{5}=4, \delta_{1}=1, \delta_{3}=1.5, \phi_{2}(t)=1+$ $t+t^{2}, \varepsilon_{1}(t)=1 / 2(1+t)^{2}, \varepsilon_{2}(t)=1 / 3(1+t)^{3}, x=1, y=1$, then plots of equation (84) are plotted in Figure 23. Type IV

$$
\begin{aligned}
\varepsilon_{1}(t) & =\int-\frac{\left(\alpha_{1}{ }^{2}+\alpha_{2}{ }^{2}\right)\left(\alpha_{1}{ }^{2} \delta_{4}{ }^{2} \phi_{2}(t)-\beta_{4}{ }^{2} \delta_{1}{ }^{2} \phi_{3}(t)\right)}{\alpha_{1}{ }^{3} \delta_{4}{ }^{2}} d t-\frac{\varepsilon_{2}(t) \alpha_{2}}{\alpha_{1}}, \varepsilon_{4}(t)=\int 2 \frac{\beta_{4}{ }^{2} \phi_{3}(t) \delta_{1}}{\alpha_{1} \delta_{4}} d t, \\
\varepsilon_{3}(t) & =\int-\frac{\alpha_{1}{ }^{2} \alpha_{3} \delta_{4}{ }^{2} \phi_{2}(t)-\beta_{4}{ }^{2} \delta_{1} \phi_{3}(t)\left(2 \alpha_{1} \delta_{3}-\alpha_{3} \delta_{1}\right)}{\alpha_{1}{ }^{2} \delta_{4}{ }^{2}} d t, \varepsilon_{5}(t)=\int-\frac{\alpha_{1}{ }^{2} \alpha_{5} \delta_{4}{ }^{2} \phi_{2}(t)-\beta_{4}{ }^{2} \delta_{1} \phi_{3}(t)\left(2 \alpha_{1} \delta_{5}-\alpha_{5} \delta_{1}\right)}{\alpha_{1}{ }^{2} \delta_{4}{ }^{2}} d t, \\
\varepsilon_{6}(t) & =\int-2 \frac{\left(\alpha_{1} \varepsilon_{2}(t)-\alpha_{2} \varepsilon_{1}(t)\right)\left(\phi_{2}(t) \alpha_{1}{ }^{2} \alpha_{2} \delta_{4}{ }^{2}-\phi_{3}(t) \alpha_{2} \beta_{4}{ }^{2} \delta_{1}{ }^{2}+\left(d / d t \varepsilon_{2}(t)\right) \alpha_{1}{ }^{2} \delta_{4}{ }^{2}\right)}{\alpha_{1}{ }^{3} \delta_{4}{ }^{2}} d t, \beta_{3}=\frac{\left(\alpha_{1} \delta_{3}-\alpha_{3} \delta_{1}\right) \beta_{4}}{\alpha_{1} \delta_{4}}, \\
\beta_{5} & =\frac{\beta_{4}\left(\alpha_{1} \delta_{5}-\alpha_{5} \delta_{1}\right)}{\alpha_{1} \delta_{4}}, \delta_{2}=\frac{\alpha_{2} \delta_{1}}{\alpha_{1}}, \phi_{4}(t)=-\frac{\beta_{4}{ }^{2} \phi_{3}(t)}{\delta_{4}{ }^{2}}, \alpha_{4}=\beta_{1}=\beta_{2}=\phi_{1}(t)=0 .
\end{aligned}
$$

The solutions are given as follows:

$$
\begin{aligned}
u_{4}= & 12 \frac{\partial^{2}}{\partial x^{2}} \ln \left(\mathbf{F}_{4}\right), \mathbf{F}_{4}=\left(\alpha_{1} x+\delta_{1} z+\varepsilon_{1}(t)\right)^{2} \\
& +\left(\alpha_{2} x+\frac{\alpha_{2} \delta_{1} z}{\alpha_{1}}+\varepsilon_{2}(t)\right)^{2}+e^{\alpha_{3} x+\left(\alpha_{1} \delta_{3}-\alpha_{3} \delta_{1}\right) \beta_{4} y / \delta_{4} \alpha_{1}+\delta_{3} z+\varepsilon_{3}(t)} \\
& +e^{\beta_{4} y+\delta_{4} z+\varepsilon_{4}(t)}+e^{\alpha_{5} x+\beta_{4}\left(\alpha_{1} \delta_{5}-\alpha_{5} \delta_{1}\right) y / \delta_{4} \alpha_{1}+\delta_{5} z+\varepsilon_{5}(t)}+\varepsilon_{6}(t)
\end{aligned}
$$

By selecting the parameters $\alpha_{1}=1, \alpha_{2}=2, \alpha_{3}=3, \alpha_{5}=5$, $\beta_{4}=3, \delta_{1}=1, \delta_{3}=1.5, \delta_{4}=2, \delta_{5}=3, \phi_{2}(t)=t, \phi_{3}(t)=t^{2}, \varepsilon_{2}$ $(t)=t^{4}, x=1, y=1$, then plots of equation (87) are plotted in Figure 24. And also, via selecting the parameters $\alpha_{1}=1, \alpha_{2}$ $=2, \alpha_{3}=3, \alpha_{5}=5, \beta_{4}=3, \delta_{1}=1, \delta_{3}=1.5, \delta_{4}=2, \delta_{5}=3, \phi_{2}$ $(t)=1, \phi_{3}(t)=2, \varepsilon_{2}(t)=3, x=1, y=1$, then plots of equation (87) are plotted in Figure 25. Moreover, via selecting the parameters $\alpha_{1}=1, \alpha_{2}=2, \alpha_{3}=3, \alpha_{5}=5, \beta_{4}=3, \delta_{1}=1, \delta_{3}$ 


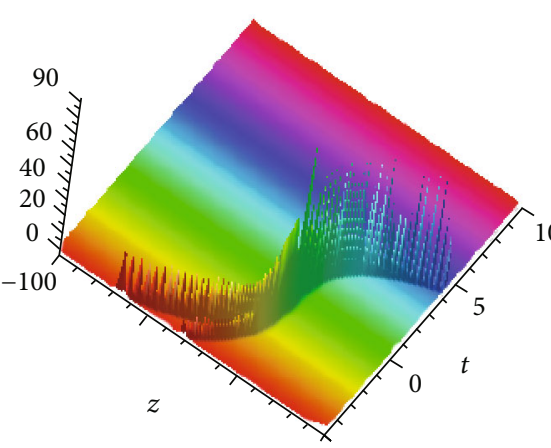

(a)

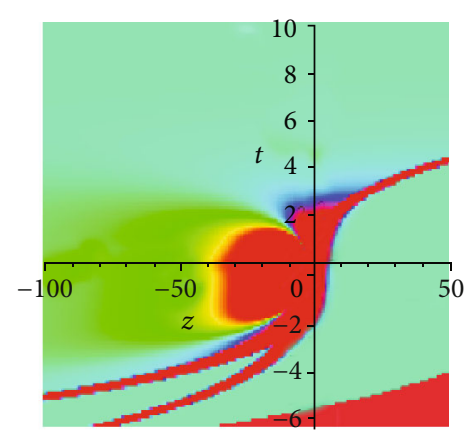

(b)

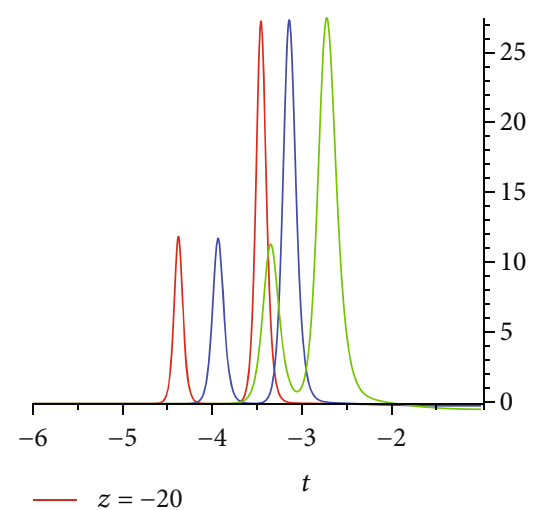

(c)

FIGURE 23: Plots of lump-three kink solutions (84).

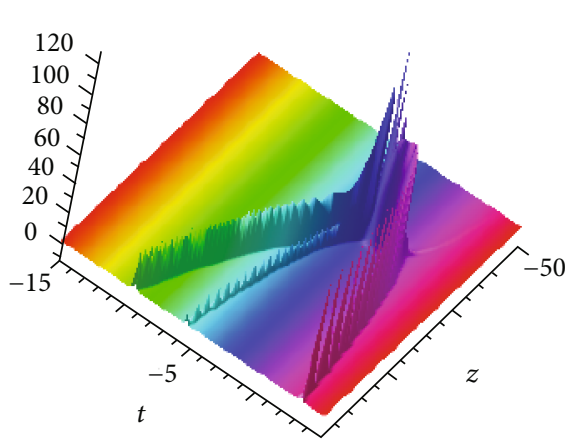

(a)

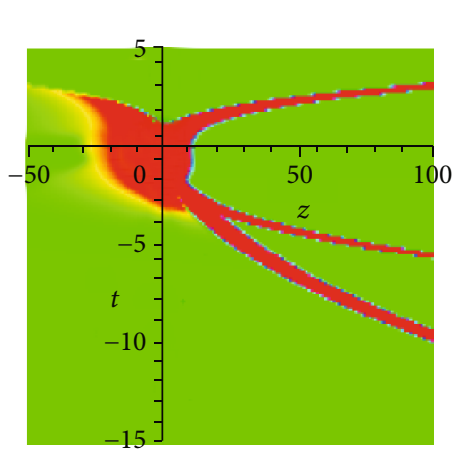

(b)

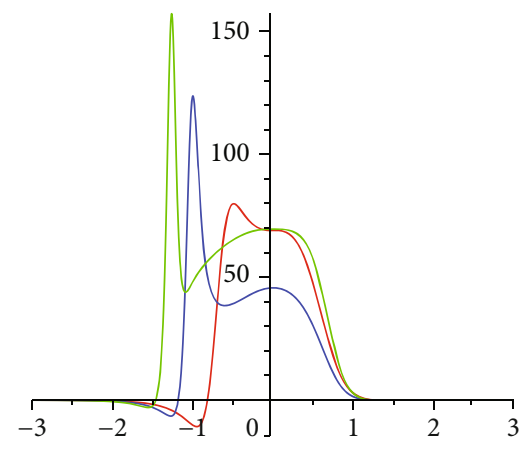

(c)

$$
\begin{aligned}
z & =0 \\
z & =1
\end{aligned}
$$

Figure 24: Plots of lump-three kink solutions (87).

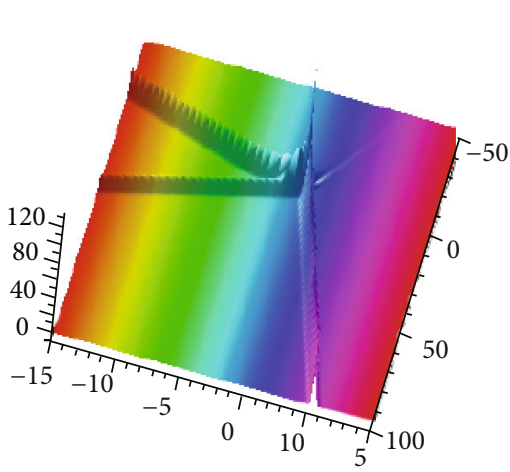

(a)

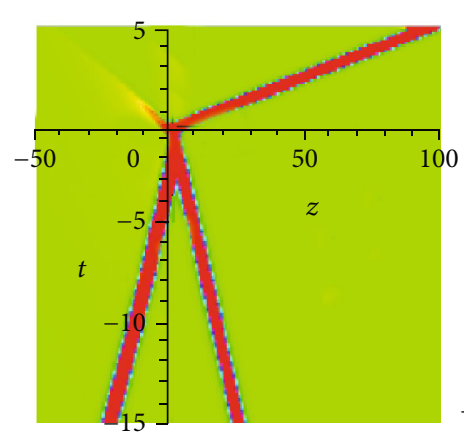

(b)

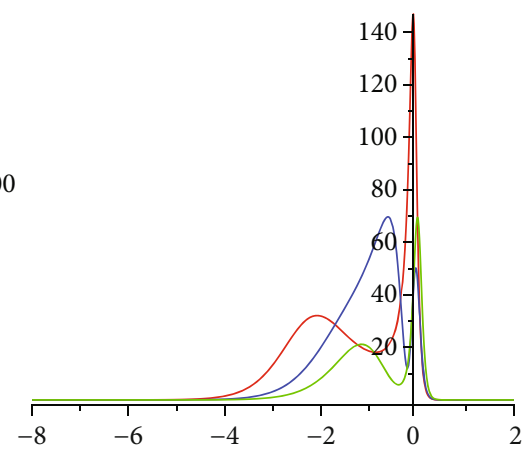

(c)

$$
\begin{aligned}
z & =-1 \\
-z & =0 \\
z & =1
\end{aligned}
$$

FIGURE 25: Plots of lump-three kink solutions (87). 


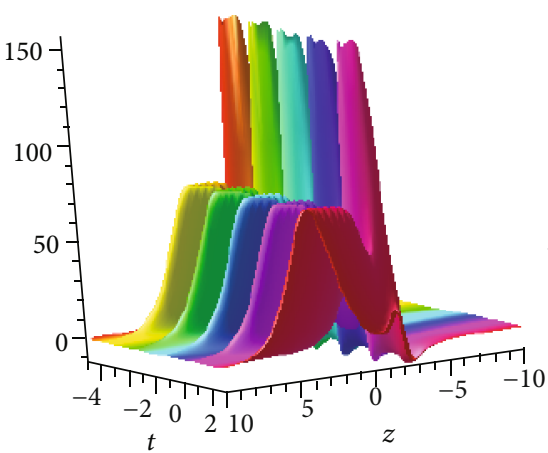

(a)

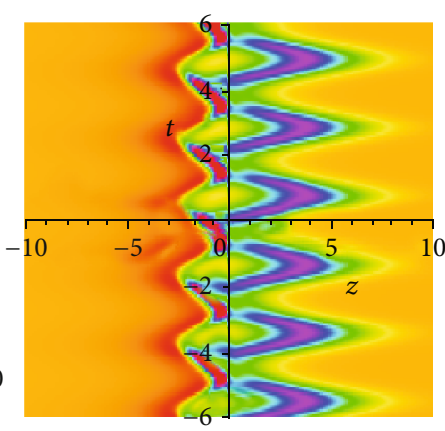

(b)

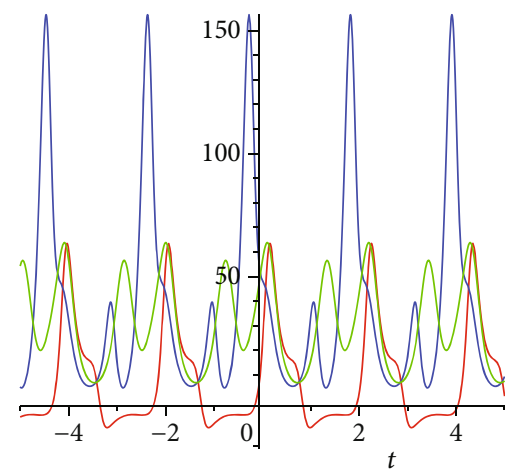

(c)

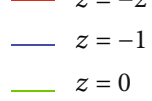

Figure 26: Plots of lump-three kink solutions (87).

$=1.5, \delta_{4}=2, \delta_{5}=3, \phi_{2}(t)=\sin (3 t), \phi_{3}(t)=\cos (3 t), \varepsilon_{2}(t)$

$=\sin (t)+\cos (t), x=1, y=1$, then plots of equation (87) are plotted in Figure 26.

\section{Conclusion}

In this paper, under the multidimensional binary Bell polynomials, we derive that the lump-soliton and its interaction solutions of the $(3+1)$-dimensional variable-coefficient nonlinear wave equation in liquid with gas bubbles has been successfully obtained. And then, ten classes for interaction between a lump-two kink solitons, three classes for interaction between two lumps, two classes for interaction between two lumps-soliton, seven classes for lump-periodic, and four classes for lump-three kink solutions were successfully constructed by employing the bilinear scheme. With the combination of the binary Bell polynomials and Hirota's bilinear method, some concrete solutions are expressed in terms of determined functions. We illustrate the regularity of solutions with some parameter constrains on constant and rational and periodic backgrounds. We also consider the classical dynamical behaviors and the elasticity of the collisions of soliton solutions and their interactions when plotting them. Finally, we conclude two dynamical behaviors of general lump-periodic and multikink soliton solutions.

\section{Data Availability}

The datasets supporting the conclusions of this article are included in the article.

\section{Conflicts of Interest}

The authors declare that they have no conflicts of interest.

\section{References}

[1] J. Manafian and M. Lakestani, “Abundant soliton solutions for the Kundu-Eckhaus equation via $\tan \left({ }_{-} \phi_{-}\left({ }_{-} \xi_{-}\right)\right)$-expansion method," Optik, vol. 127, no. 14, pp. 5543-5551, 2016.
[2] M. Dehghan and J. Manafian, "The solution of the variable coefficients fourth-order parabolic partial differential equations by the homotopy perturbation method," Zeitschrift für Naturforschung A, vol. 64, no. 7-8, pp. 420-430, 2009.

[3] E. Alimirzaluo, M. Nadjafikhah, and J. Manafian, "Some new exact solutions of $(3+1)$-dimensional Burgers system via Lie symmetry analysis," Advances in Difference Equations, vol. 2021, no. 1, 2021.

[4] X. H. Zhao, B. Tian, X. Y. Xie, X. Y. Wu, Y. Sun, and Y. J. Guo, "Solitons, Bäcklund transformation and Lax pair for a $(2+1)$ dimensional Davey-Stewartson system on surface waves of finite depth," Waves in Random and Complex Media, vol. 28, no. 2, pp. 356-366, 2018.

[5] M. R. A. Fahim, P. R. Kundu, M. E. Islam, M. A. Akbar, and M. S. Osman, "Wave profile analysis of a couple of $(3+1)$ dimensional nonlinear evolution equations by sine-Gordon expansion approach," Journal of Ocean, Engineering and Science, 2021.

[6] I. Siddique, M. M. M. Jaradat, A. Zafar, K. B. Mehdi, and M. S. Osman, "Exact traveling wave solutions for two prolific conformable M-fractional differential equations via three diverse approaches," Results in Physics, vol. 28, article 104557, 2021.

[7] R. Yao, Y. Li, and S. Lou, "A new set and new relations of multiple soliton solutions of $(2+1)$-dimensional Sawada-Kotera equation," Communications in Nonlinear Science and Numerical Simulation, vol. 99, article 105820, 2021.

[8] Y. L. Ma, A. M. Wazwaz, and B. Q. Li, "A new (3+1)-dimensional Kadomtsev-Petviashvili equation and its integrability, multiple-solitons, breathers and lump waves," Mathematics and Computers in Simulation, vol. 187, pp. 505-519, 2021.

[9] X. Guan and W. Liu, "Multiple-soliton and lump-kink solutions for a generalized (3+1)-dimensional KadomtsevPetviashvili equation," Results in Physics, vol. 17, article 103149, 2020.

[10] X. Hong, A. I. A. Alkireet, O. A. Ilhan, J. Manafian, and M. K. M. Nasution, "Multiple soliton solutions of the generalized Hirota-Satsuma-Ito equation arising in shallow water wave," Journal of Geometry and Physics, vol. 170, article 104338, 2021.

[11] J. Manafian and M. Lakestani, "N-lump and interaction solutions of localized waves to the $(2+1)$-dimensional variable-coefficient Caudrey-Dodd-Gibbon-Kotera-Sawada equation," Journal of Geometry and Physics, vol. 150, article 103598, 2020. 
[12] S. T. R. Rizvi, A. R. Seadawy, S. Ahmed, M. Younis, and K. Ali, "Study of multiple lump and rogue waves to the generalized unstable space time fractional nonlinear Schrodinger equation," Chaos, Solitons \& Fractals, vol. 151, article 111251, 2021.

[13] N. Abdeljalil, N. Mourad, and B. Gasimov, "Parallel numerical computation of an analytical method for solving an inverse problem," Advanced Mathematical Models \& Applications, vol. 6, no. 2, pp. 162-173, 2021.

[14] S. T. R. Rizvi, A. R. Seadawy, M. Younis, K. Ali, and H. Iqbal, "Lump-soliton, lump-multisoliton and lump-periodic solutions of a generalized hyperelastic rod equation," Modern Physics Letters B, vol. 35, no. 11, article 2150188, 2021.

[15] W. X. Ma, "Lump solutions to the Kadomtsev-Petviashvili equation," Physics Letters A, vol. 379, no. 36, pp. 1975-1978, 2015.

[16] Z. Li, J. Manafian, N. Ibrahimov, A. Hajar, K. S. Nisar, and W. Jamshed, "Variety interaction between _ $\mathrm{k}_{\text {_ }}$-lump and _ $\mathrm{k}_{\text {_ }}$-kink solutions for the generalized Burgers equation with variable coefficients by bilinear analysis," Results in Physics, vol. 28, article 104490, 2021.

[17] Y. Xiao, E. Fan, and P. Liu, "Inverse scattering transform for the coupled modified Korteweg-de Vries equation with nonzero boundary conditions," Journal of Mathematical Analysis and Applications, vol. 504, no. 2, article 125567, 2021.

[18] X. Zhang and Y. Chen, "Inverse scattering transformation for generalized nonlinear Schrodinger equation," Applied Mathematics Letters, vol. 98, pp. 306-313, 2019.

[19] Q. Shi and C. Li, "Darboux transformations of the supersymmetric constrained B and C type KP hierarchies," Journal of Geometry and Physics, vol. 165, article 104216, 2021.

[20] J. Rao, J. He, D. Mihalache, and Y. Cheng, "Dynamics and interaction scenarios of localized wave structures in the Kadomtsev-Petviashvili-based system," Applied Mathematics Letters, vol. 94, pp. 166-173, 2019.

[21] L. Li, C. Duan, and F. Yu, "An improved Hirota bilinear method and new application for a nonlocal integrable complex modified Korteweg-de Vries (MKdV) equation," Physics Letters A, vol. 383, no. 14, pp. 1578-1582, 2019.

[22] Y. L. Sun, W. X. Ma, and J. P. Yu, "N-soliton solutions and dynamic property analysis of a generalized three- component Hirota-Satsuma coupled KdV equation," Applied Mathematics Letters, vol. 120, article 107224, 2021.

[23] W. X. Ma, "N-soliton solution and the Hirota condition of a (2 +1)-dimensional combined equation," Mathematics and Computers in Simulation, vol. 190, pp. 270-279, 2021.

[24] P. Cui, "Bilinear form and exact solutions for a new extended $(2+1)$-dimensional Boussinesq equation," Results in Physics, vol. 22, article 103919, 2021.

[25] X. Cheng, S. Lou, Y. Yang, P. Li, and S. Qi, “The N-soliton molecule for the combined $(2 \mathrm{~N}+1)$ th-order Lax's KdV equation," Results in Physics, vol. 18, article 103184, 2020.

[26] J. Manafian, "Novel solitary wave solutions for the $(3+1)$ dimensional extended Jimbo-Miwa equations," Computers \& Mathematics with Applications, vol. 76, no. 5, pp. 1246-1260, 2018.

[27] W. X. Ma, "A search for lump solutions to a combined fourth order nonlinear PDE in (2+1)-dimensions," Journal of Applied Analysis and Computation, vol. 9, no. 4, pp. 1319-1332, 2019.

[28] W. X. Ma, "Interaction solutions to Hirota-Satsuma-Ito equation in (2+1)-dimensions," Frontiers of Mathematics in China, vol. 14, no. 3, pp. 619-629, 2019.
[29] G. F. Deng and Y. T. Gao, "Integrability, solitons, periodic and travelling waves of a generalized $(3+1)$-dimensional variablecoefficient nonlinear-wave equation in liquid with gas bubbles," European Physical Journal Plus, vol. 132, no. 6, pp. 255-271, 2017.

[30] J. G. Liu, W. H. Zhu, Y. He, and Y. K. Wu, "Interaction phenomena between lump and solitary wave of a generalized (3 +1 )-dimensional variable-coefficient nonlinear-wave equation in liquid with gas bubbles," Communications in Theoretical Physics, vol. 72, no. 8, article 085002, 2020.

[31] Y. R. Guo and A. H. Chen, "Hybrid exact solutions of the (3+ 1)-dimensional variable-coefficient nonlinear wave equation in liquid with gas bubbles," Results in Physics, vol. 23, article 103926, 2021.

[32] H. Wang, S. Tian, T. Zhang, and Y. Chen, "Lump wave and hybrid solutions of a generalized (3+1)-dimensional nonlinear wave equation in liquid with gas bubbles," Frontiers of Mathematics in China, vol. 14, no. 3, pp. 631-643, 2019.

[33] Y. R. Guo and A. H. Chen, "Families of exact solutions of the generalized (3+1)-dimensional nonlinear-wave equation," Modern Physics Letters B, vol. 32, no. 29, article 1850359, 2018.

[34] W. Liu and Y. Zhang, "Resonant multiple wave solutions, complexiton solutions and rogue waves of a generalized (3 +1 )-dimensional nonlinear wave in liquid with gas bubbles," Waves in Random and Complex Media, vol. 30, no. 3, pp. 470-480, 2020.

[35] W. X. Ma, "N-soliton solutions and the Hirota conditions in (2 +1)-dimensions," Optical and Quantum Electronics, vol. 52, no. 12, article 511, 2020.

[36] M. F. Uddin, M. G. Hafez, Z. Hammouch, and D. Baleanu, "Periodic and rogue waves for Heisenberg models of ferromagnetic spin chains with fractional beta derivative evolution and obliqueness," Waves in Random and Complex Media, pp. 1$15,2020$.

[37] A. Houwe, J. Sabi'u, Z. Hammouch, and S. Y. Doka, "Solitary pulses of a conformable nonlinear differential equation governing wave propagation in low-pass electrical transmission line," Physics Scripta, vol. 95, no. 4, article 045203, 2020.

[38] O. A. Ilhan, J. Manafian, H. M. Baskonus, and M. Lakestani, "Solitary wave solitons to one model in the shallow water waves," European Physical Journal Plus, vol. 136, no. 3, article 337, 2021.

[39] M. F. Uddin, M. G. Hafez, Z. Hammouch, H. Rezaadeh, and D. Baleanu, "Traveling wave with beta derivative spatialtemporal evolution for describing the nonlinear directional couplers with metamaterials via two distinct methods," Alexandria Engineering Journal, vol. 60, no. 1, pp. 1055-1065, 2021.

[40] K. Hosseini, M. Samavat, M. Mirzazadeh, W. X. Ma, and Z. Hammouch, "A new (3+1)-dimensional Hirota bilinear equation: its Bäcklund transformation and rational-type solutions," Regular and Chaotic Dynamics, vol. 25, no. 4, pp. 383391, 2020.

[41] M. G. Hafez, S. A. Iqbal, and Z. Hammouch, "Dynamical behaviors and oblique resonant nonlinear waves with dualpower law nonlinearity and conformable temporal evolution," Discrete \& Continuous Dynamical Systems-S, vol. 14, no. 7, pp. 2245-2260, 2021.

[42] H. F. Ismael, H. Bulut, H. M. Baskonus, and W. Gao, "Dynamical behaviors to the coupled Schrödinger-Boussinesq system with the beta derivative," AIMS Mathematics, vol. 6, no. 7, pp. 7909-7928, 2021. 
[43] G. Yel, C. Cattani, H. M. Baskonus, and W. Gao, "On the complex simulations with dark-bright to the Hirota-Maccari system," Journal of Computational and Nonlinear Dynamics, vol. 16, no. 6, article 061005, 2021.

[44] L. T. Gai, W. X. Ma, and M. C. Li, "Lump-type solution and breather lump-kink interaction phenomena to a (\$\$ mathbf $\{\{3\{\backslash$ varvec $\{+\}\} 1\}\} \$$ )-dimensional GBK equation based on trilinear form," Nonlinear Dynamics, vol. 100, no. 3, pp. 2715-2727, 2020.

[45] W. X. Ma, "Bilinear equations, Bell polynomials and linear superposition principle," Journal of Physics: Conference Series, vol. 411, article 012021, 2013. 